\title{
Observable spectral and angular distributions of $\gamma$-rays from extragalactic ultrahigh energy cosmic ray accelerators: the case of extreme TeV blazars
}

\author{
Emil V. Khalikov, ${ }^{1 \star}$ Timur A. Dzhatdoev ${ }^{1,2} \dagger$ \\ ${ }^{1}$ Federal State Budget Educational Institution of Higher Education, M.V. Lomonosov Moscow State University, \\ Skobeltsyn Institute of Nuclear Physics (SINP MSU), 1(2), Leninskie gory, GSP-1, 119991 Moscow, Russia \\ ${ }^{2}$ Institute for Cosmic Ray Research, University of Tokyo, 5-1-5 Kashiwanoha, 277-8568 Kashiwa, Japan
}

Accepted XXX. Received YYY; in original form ZZZ

\begin{abstract}
Ultrahigh energy protons and nuclei from extragalactic cosmic ray sources initiate intergalactic electromagnetic cascades, resulting in observable fluxes of $\gamma$-rays in the GeV-TeV energy domain. The total spectrum of such cascade $\gamma$-rays of hadronic nature is significantly harder than the one usually expected from blazars. The spectra of some sources known as "extreme TeV blazars" could be well-described by this "intergalactic hadronic cascade model" (IHCM). We calculate the shape of the observable point-like spectrum, as well as the observable angular distibution of $\gamma$-rays, for the first time taking into account the effect of primary proton deflection in filaments and galaxy clusters of the extragalactic magnetic field assuming the model of Dolag et al. (2005). We present estimates of the width of the observable $\gamma$-ray angular distribution derived from simple geometrical considerations. We also employ a hybrid code to compute the observable spectral and angular distributions of $\gamma$-rays. The observable point-like spectrum at multi-TeV energies is much softer than the one averaged over all values of the observable angle. The presence of a high-energy cutoff in the observable spectra of extreme TeV blazars in the framework of the IHCM could significantly facilitate future searches of new physics processes that enhance the apparent $\gamma$-ray transparency of the Universe (for instance, $\gamma \rightarrow A L P$ oscillations). The width of the observable angular distribution is greater than or comparable to the extent of the point spread function of next-generation $\gamma$-ray telescopes.
\end{abstract}

Key words: astroparticle physics - radiation mechanisms: non-thermal - galaxies: active (galaxies:) BL Lacertae objects: individual: 1ES 1101-232 - (galaxies:) BL Lacertae objects: individual: 1ES 0347-121 - (galaxies:) BL Lacertae objects: individual: 1ES 0229+200

\section{INTRODUCTION}

Observations of ultrahigh energy cosmic rays (UHECR) indicate that these particles are mostly extragalactic, at least when the primary particle energy $E_{0}$ exceeds $10^{19} \mathrm{eV}=10 \mathrm{EeV}$ (Aab et al. $2017 \mathrm{~b})^{1}$. Active galactic nuclei (AGN) and, in particular, blazars (AGN with the jets presumably pointed towards the observer) are among the most well-motivated sources of UHECR (Hillas 1984; Biermann \& Strittmatter 1987; Rachen \& Biermann 1993; Dermer \& Razzaque 2010).

Ultrahigh energy protons and nuclei from extragalactic sources interact with diffuse photon backgrounds by

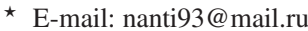

$\dagger$ E-mail: timur1606@gmail.com

1 see, however, Gruzinov (2018) and Strong (2018)
}

means of photohadronic processes and electron-positron pair production (Greisen 1966; Zatsepin \& Kuz'min 1966; Berezinskii \& Grigor'eva 1988; Berezinsky et al. 2006)². Secondary $\gamma$-rays, electrons and positrons (hereafter "electrons" for simplicity) develop intergalactic electromagnetic (EM) cascades (Berezinsky \& Smirnov 1975; Protheroe 1986; Protheroe \& Stanev 1993; Aharonian et al. 1994; Berezinsky \& Kalashev 2016) via the pair production (PP) process $\left(\gamma \gamma \rightarrow e^{+} e^{-}\right)$and the inverse Compton (IC) scattering $\left(e^{+} \gamma \rightarrow e^{+^{\prime}} \gamma^{\prime}\right.$ or $\left.e^{-} \gamma \rightarrow e^{-^{\prime}} \gamma^{\prime}\right)$. Cascade $\gamma$-rays that typically have the energy much lower than that of the primary protons may be observed with space $\gamma$-ray telescopes such as Fermi-LAT (Atwood et al. 2009), AGILE (Tavani et al. 2009; Bulgarelli et al. 2019) or projected space observatories MAST (Dzhatdoev \& Podlesnyi 2019), GAMMA-400 (Galper et al. 2013),

\footnotetext{
2 in what follows we will mainly consider the case of primary protons
} 
e-ASTROGAM (De Angelis et al. 2018), AMEGO (McEnery et al. 2019), AdEPT (Hunter et al. 2014), HARPO (Gros et al. 2018), or with ground-based $\gamma$-ray detectors such as H.E.S.S. (Hinton 2004; Bonnefoy et al. 2018), MAGIC (Aleksić et al. 2016a,b), VERITAS (Krennrich et al. 2004; Park 2016), HAWC (Abeysekara et al. 2017), Cherenkov Telescope Array (CTA) (Actis et al. 2011; Acharya et al. 2013; The CTA Consortium 2018), ASTRI (Lombardi et al. 2020), LHAASO (Cui et al. 2014; Tian et al. 2018; Bai et al. 2019).

The observable angular distribution of these cascade $\gamma$-rays from a point-like source strongly depends on the strength and structure of the extragalactic magnetic field (EGMF). In particular, if the deflection angles of primary protons and cascade electrons are small enough, the observational appearance of the source is similar to a slightly extended $\gamma$-ray pattern. The idea that cascade $\gamma$ rays from primary protons accelerated in extragalactic sources may contribute to point-like images of these sources was considered in Waxman \& Coppi (1996) and Uryson (1998), thus laying the foundation for the "intergalactic hadronic cascade model" (IHCM). Waxman \& Coppi (1996) mainly concentrated on temporal properties of the $\gamma$-ray signal and a general discussion of the IHCM, while Uryson (1998) demonstrated that a part of the observable $\gamma$-rays may have a multi-TeV energy. The appearance of these very energetic $\gamma$-rays is due to the fact that a part of the interactions of the primary protons occurs relatively near to the observer, and thus the cascade $\gamma$-rays experience a lesser degree of absorption on diffuse extragalactic photon backgrounds than the primary $\gamma$-rays from the same source. $\gamma$-ray spectra of EM cascades initiated by UHECR injected at cosmological distances were calculated in (Kalashev et al. 2001, 2009).

However, in $\gamma$-ray astronomy it is still customary to neglect any effects from intergalactic EM cascades initiated by either $\gamma$ rays or protons, assuming the so-called "absorption-only model" (AOM) that accounts for only PP and adiabatic losses. In the framework of the AOM one can reconstruct the shape of the intrinsic spectrum of the source by compensating for the attenuation of the primary $\gamma$-ray flux and redshift. Observations made with imaging atmospheric Cherenkov telescopes (IACTs) revealed that some blazars have such reconstructed intrinsic spectral energy distributions (SEDs) peaking at $E>1 \mathrm{TeV}$ (e.g. Aharonian et al. (2006); Aliu et al. (2014)). In what follows we call these AGNs "extreme TeV blazars" (ETBs).

ETBs are defined solely by their $\gamma$-ray properties, but they have much in common with extreme highly peaked BL Lac objects (EHBLs) (Costamante et al. 2001; Bonnoli et al. 2015; Costamante et al. 2018), which may be characterised by their broadband (in particular, X-ray) properties. Indeed, some blazars such as 1ES 0229+200 (Aharonian et al. 2007c; Aliu et al. 2014), 1ES 1101-232 (Aharonian et al. 2006, 2007a) and 1ES 0347-121 (Aharonian et al. 2007b) may be classified as both ETBs (see Dzhatdoev et al. (2017), Fig. 12, 14, 17) and EHBLs. Compared to well-known nearby blazars such as Mkn 501 and Mkn 421, ETBs, as a rule, reveal weak and slow variability in the high energy (HE, $E>100 \mathrm{MeV}$ ) and the very high energy (VHE, $E>100 \mathrm{GeV}$ ) spectral bands.

These peculiar properties of ETBs allow for a possibility that a significant part of observable $\gamma$-rays were in fact produced not inside the source, but as a result of EM cascade development in the intergalactic medium. Therefore, the IHCM experienced a very high level of popularity when applied to ETBs and EHBLs (Essey et al. 2010, 2011; Murase et al. 2012; Zheng et al. 2015; Archer et al.
2018; Acciari et al. 2019b; Acciari et al. 2020; Das et al. 2020); for a review see (Biteau et al. 2020).

Even before the discovery of ETBs in the VHE energy range, first sophisticated models of the EGMF in the largescale structure (LSS) were developed (Dolag et al. 2005; Sigl et al. 2004; Das et al. 2008; Vazza et al. 2017; Alves Batista et al. 2017; Hackstein et al. 2018, 2019). Most of these models predict an appreciable $\left(\sim 0.1-1^{\circ}\right)$ deflection for UHE protons after propagating $\sim 100 \mathrm{Mpc}$, and the EGMF appears to be strongly inhomogeneous (Ryu et al. 1998; Medina Tanco 1998), with relatively strong magnetic fields in galaxy clusters $(B \sim 1-10 \mu \mathrm{G}), B \sim 1-100 \mathrm{nG}$ in filaments, and comparatively weak $(B<1 \mathrm{nG})$ EGMF in voids. There is mounting evidence for the existence of magnetic fields in extragalactic filaments. In particular, Govoni et al. (2019) report the detection of synchrotron emission from a filament connecting two galaxy clusters (see also Vacca et al. (2018), where the detection of another filament of the cosmic web was reported).

In the present paper, for the first time, we show how primary proton deflections in extragalactic magnetic field described by large scale structure formation models (in particular, strongly inhomogeneous EGMF) modify the observable spectral and angular distributions of $\gamma$-rays. We demonstrate that this effect broadens the observable angular distribution significantly and leads to an effective cutoff in the observable point-like spectrum ${ }^{3}$ (in what follows called simply "observable spectrum"). Some preliminary estimates justifying these conclusions were published in our recent work Dzhatdoev et al. (2019) as conference contribution. The deflection of cascade electrons in weaker magnetic fields of LSS voids may contribute to the broadening of the observable angular distribution, leading to an even more dramatic effect.

The present work was in part motivated by the ongoing search for oscillations of $\gamma$-rays to axion-like particles $(\gamma \rightarrow A L P)$ using blazar spectra (Horns \& Meyer 2012; Rubtsov \& Troitsky 2014; Dzhatdoev 2015; Korochkin et al. 2019). $\gamma$-rays may convert to ALPs (Raffelt \& Stodolsky 1988; De Angelis et al. 2007; Kartavtsev et al. 2017; Montanino et al. 2017; Galanti \& Roncadelli 2018; Galanti et al. 2019) in magnetic fields of the source or in the EGMF clusters or filaments; the produced ALPs then may convert back to observable $\gamma$-rays. A part of the path of these observable $\gamma$-rays is, thus, traversed by ALPs that do not experience absorption on extragalactic background light (EBL) photons; therefore, the optical depth $\tau$ for $\gamma$-rays is effectively reduced and the Universe appears more transparent in the presence of the $\gamma \rightarrow A L P$ oscillation process.

The discovery of ALPs by means of this effect would require a detailed estimation of astrophysical backgrounds for the $\gamma \rightarrow A L P$ process and then a suppression of these backgrounds. Cascade $\gamma$ rays from primary proton interactions in fact represent the most dangerous source of the background for these $\gamma \rightarrow A L P$ oscillation searches (Baklagin et al. 2018). The presence of an effective cutoff in the observable spectrum with respect to the angle-averaged spectrum in the framework of the IHCM allows to greatly reduce the background for $\gamma \rightarrow A L P$ oscillation search in blazar spectra at high values of the $\gamma \gamma$ opacity.

This paper is organized as follows. In Sect. 2 we present some basic estimates of the angular broadening and spectral cutoff effects, devoid of any unnecessary computational details. In Sect. 3 we describe simulations of proton deflection and calculation of the

3 that is, the spectrum inside the point spread function (PSF) of the observing instrument 
observable spectrum by means of a hybrid method introduced by us in Dzhatdoev et al. (2017) (hereafter denoted as D17). We present the main results of this paper in Sect. 4 and observational prospects - in Sect. 5. We briefly discuss these results and conclude in Sect. 7.

Throughout this work we assume the following values of cosmological parameters: $H_{0}=67.8 \mathrm{~km} \mathrm{~s}^{-1} \mathrm{Mpc}^{-1}, \Omega_{m}=0.308$, $\Omega_{\Lambda} \approx 1-\Omega_{m}$ (Ade et al. 2016). Somewhat different results for these parameters were presented in Aghanim et al. (2018): $H_{0}=67.4 \mathrm{~km}$ $\mathrm{s}^{-1} \mathrm{Mpc}^{-1}$ and $\Omega_{m}=0.315$; these updated values, however, would not influence our results and conclusions significantly.

\section{ESTIMATES}

Primary protons deflect to appreciable angles while they propagate over a large distance $L_{S}>100 \mathrm{Mpc}$ from the source to the observer. According to the EGMF model of Dolag et al. (2005) (hereafter denoted as D05), a half of protons with the energy $E_{p}=40 \mathrm{EeV}$ deflect to the angle $\delta>0.7^{\circ}$ for $L_{S}=500 \mathrm{Mpc}$ (see Fig. 15 in the aforementioned paper). For larger distances, neglecting proton energy losses, we estimate

$$
0.7^{\circ} \sqrt{\frac{L_{S}}{500 M p c}} \frac{40 \mathrm{EeV}}{E_{p}}<\delta<0.7^{\circ} \frac{L_{S}}{500 M p c} \frac{40 \mathrm{EeV}}{E_{p}}
$$

in particular, for $E_{p}=30 \mathrm{EeV}$ and $z_{s}=0.1861 .2^{\circ}<\delta<1.5^{\circ}$ (throughout this section we conservatively assume $\delta=1^{\circ}$ ). ${ }^{4}$ The total value of $\delta$ may be dominated by one or two of the most prominent magnetic structures, or may accumulate over many less significant ones. Below we estimate the width of the observable $\gamma$-ray angular distribution separately for these cases.

In the present work we concentrate on the impact that primary proton deflections have on the observable spectral and angular distributions of $\gamma$-rays. Cascade electrons also experience deflections on magnetic fields of filaments and voids. The electrons that were produced in filaments are typically almost completely isotropized (their deflection angles $\delta_{e} \gg 1 \mathrm{rad}$ ), and thus the secondary (cascade) $\gamma$-rays form a quasi-isotropic cloud (the so-called "pair halo", PH), as discussed in Aharonian et al. (1994). Such PHs are typically difficult to find in the spectra of highly beamed sources such as blazars (Aharonian et al. 2001); therefore, we do not consider this component of observable $\gamma$-rays. The account of PHs would render the observable angular distribution even broader than in the framework of our model and thus would reinforce our conclusions. We also neglect synchrotron losses (see Aharonian et al. (2010) and Fig. 9 of Berezinsky \& Kalashev (2016) for related discussions).

The constraints on the strength of the EGMF in voids are being actively debated (Neronov \& Vovk 2010; Tavecchio et al. 2010; Dermer et al. 2011; Taylor et al. 2011; Arlen et al. 2014; Finke et al. 2015; Ackermann et al. 2018a); no reliable measurement of these magnetic fields is available by now. The typical energy loss length of cascade electrons is much smaller than the extension of the void (see Dzhatdoev et al. (2019), Fig. 2). ${ }^{5}$ Depending on the strength and the correlation length of the EGMF in voids, cascade $\gamma$-rays may form a PH or, in case of small electron deflection angle $\delta_{e} \ll 1 \mathrm{rad}$, appear as a highly anisotropic "magnetically broadened cascade" (MBC) pattern (Abramowski et al. 2014).

\footnotetext{
${ }^{4}$ the account of energy losses would increase the value of $\delta$

5 this figure was produced assuming the approximation for the IC process presented in Khangulyan et al. (2014)
}

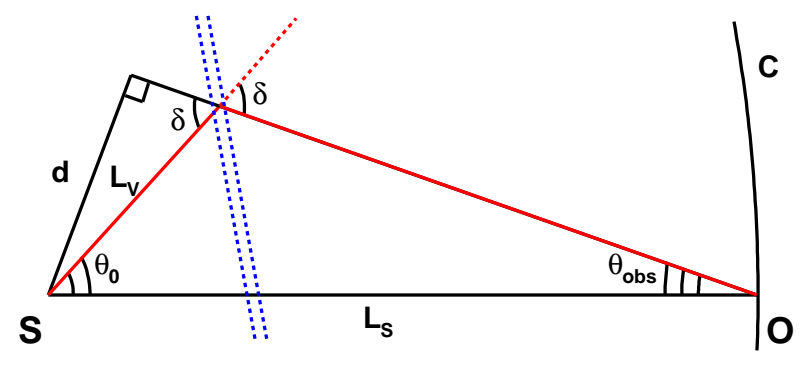

Figure 1. Geometry scheme for the intergalactic hadronic cascade model and one filament (not to scale, see text for more details).

\subsection{Individual effect}

Here we consider the case where the total deflection is due to one filament of the $\mathrm{LSS}^{6}$. A simplified scheme of the corresponding geometry is shown in Fig. 1. A source $(S)$ emits UHE protons that first propagate through a void (underdense region of space) with the diameter $L_{V}$ and then are upscattered on a filament (denoted by twin dashed blue lines; the deflection angle is, again, denoted as $\delta)$. An example of a proton path is shown as a red line $\left(\theta_{0}\right.$ is the primary proton emission angle). The observer $(O)$, located at a distance $L_{S}$ from the source, detects secondary $\gamma$-rays from cascades initiated by the proton. Primary protons are deflected by the Galactic magnetic field and are not registered from the same direction as the $\gamma$-rays.

We extrapolate the observable $\gamma$-ray path until we have a triangle with a right angle. The side $d$ of that triangle can be represented by the following equation:

$$
d=L_{V} \sin (\delta)=L_{S} \sin \left(\theta_{\text {obs }}\right),
$$

where $\theta_{o b s}$ is the observable angle (the angle between the direction from the source to the observer and the direction of the incoming observable $\gamma$-ray $)^{7}$. Therefore, $\theta_{o b s}$ could be estimated as follows:

$$
\sin \left(\theta_{o b s}\right)=\sin (\delta) \frac{L_{V}}{L_{S}} .
$$

We note that the same expression is widely known for purely electromagnetic cascades (Neronov \& Semikoz 2009); the physical interpretation of the quantities entering into this expression is, however, very different.

Following Harari et al. (2016), we estimate a typical astrophysically plausible value of the deflection angle on a single filament as

$$
\delta \approx 1^{\circ} \frac{B}{1 n G} \frac{40 E e V}{E_{p} / Z} \frac{\sqrt{L_{B} l_{c}}}{1 M p c},
$$

where $B$ is the magnetic field strength, $Z$ is the charge number of the primary particle (proton in our case), $L_{B}$ is the thickness of the filament and $l_{c}$ is the correlation length for that magnetic field. For $B=1 \mathrm{nG}, L_{B}=1 \mathrm{Mpc}, l_{c}=1 \mathrm{Mpc}$, we get $\delta=1^{\circ}$ for $E=40$ $\mathrm{EeV}$ and $\delta=1.33^{\circ}$ for $E=30 \mathrm{EeV}$. Our estimates of $\theta_{\text {obs }}$ for the blazar 1ES 1101-232 $\left(z_{s}=0.186\right), \delta=1^{\circ}$ and several values of $L_{V}$ are presented in Table 1 (first line). The typical extension of an IACT PSF is about $0.1^{\circ}$, thus, a part of observable $\gamma$-ray flux in our case will not fit into the point-like image of the source.
6 deflections on galaxy clusters are usually important for large redshifts, $z_{s}>0.3-0.5$ (see Subsection 6.2 in D05)

7 for $\gamma$-rays produced after the proton was deflected on the filament 
Table 1. Estimates of the observable angle $\theta_{o b s}\left[{ }^{\circ}\right]$ for models with different number of filaments.

\begin{tabular}{|c|cccc|}
\hline$L_{V} / L_{V_{2}} / L$ & $50 \mathrm{Mpc}$ & $100 \mathrm{Mpc}$ & $200 \mathrm{Mpc}$ & $500 \mathrm{Mpc}$ \\
\hline one filament & 0.064 & 0.13 & 0.25 & 0.64 \\
two filaments & 0.073 & 0.16 & 0.37 & 2.1 \\
n filaments & 0.016 & 0.045 & 0.13 & 0.51 \\
\hline
\end{tabular}

Below, in Sect. 4, we present results for a specific case of the isotropic angular distribution of protons. This greatly simplifies calculations, as all observable $\gamma$-ray events are collected from the observer sphere $C$.

\subsection{Collective effect}

Now let us assume that the primary protons go through two filaments before reaching the observer (see Fig. 2). The notations here are mostly the same as in Subsect. 2.1, but we consider the specific case of $\theta_{0}=0$ and introduce another void between the filaments with the diameter $L_{V_{2}}$ (the diameter of the first void is now denoted as $L_{V_{1}}$ ).

In analogy with eq. $(2)^{8}$ :

$$
d=\left(L_{S}-L_{V_{1}}\right) \sin \left(\theta_{o b s}\right)=L_{V_{2}} \sin \left(\frac{\pi}{2}-\alpha\right)
$$

At the same time

$$
\delta+\alpha=\frac{\pi}{2}-\theta_{o b s} \rightarrow \frac{\pi}{2}-\alpha=\delta+\theta_{o b s}
$$

Assuming that $\theta_{o b s} \ll \frac{\pi}{2}$ and $\delta \ll \frac{\pi}{2}$ we obtain the following:

$$
\left(L_{S}-L_{V_{1}}\right) \theta_{o b s} \approx L_{V_{2}}\left(\delta+\theta_{o b s}\right) \text {, }
$$

and finally

$$
\theta_{o b s} \approx \frac{\delta L_{V_{2}}}{L_{S}-L_{V_{1}}-L_{V_{2}}} .
$$

Assuming $L_{V_{1}}=50 \mathrm{Mpc}$ and various values of $L_{V_{2}}$, we estimate $\theta_{\text {obs }}$ (see second line of Table 1). Finally, we consider the case of many filaments $n=L_{S} / L_{V} \gg 1$ when the total deflection of the primary proton $\delta \sim \delta_{n} \sqrt{n}$. For cascades initiated at the distance $L$ from the source:

$$
\theta_{o b s} \sim \delta_{n} \sqrt{\frac{L}{L_{V}}} \frac{L}{L_{S}}=\delta\left(\frac{L}{L_{S}}\right)^{3 / 2} .
$$

Numerical estimates of $\theta_{o b s}$ for this case are also presented in Table 1 (third line).

\subsection{EM cascade spectral features}

The shape of the observable spectrum of an EM cascade depends on the distance between its origin and the observer. SEDs of cascades initiated by $\gamma$-rays with the energy $E_{0}=1 \mathrm{PeV}$ are shown in Fig. 3 for various ranges of their origin redshift $z_{0}$. These calculations were made with the ELMAG 2.03 publiclyavailable code (Kachelrieß et al. 2012). The distribution on $z_{0}$ is random with a uniform probability density function. In Sect. 4-5 of this work we present results for the two following EBL models: Kneiske \& Dole (2010) (hereafer KD10) and Gilmore et al. (2012) (hereafer G12); other options include Primack et al. (2005); Franceschini et al. (2008); Domínguez et al. (2011); Inoue et al.

\footnotetext{
${ }^{8}$ here we consider $\gamma$-rays produced after the proton had undergone two deflections
}

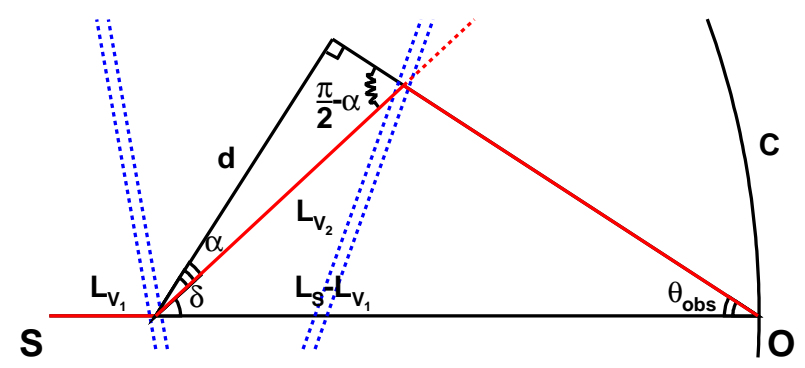

Figure 2. Geometry scheme for the intergalactic hadronic cascade model and two filaments (not to scale).

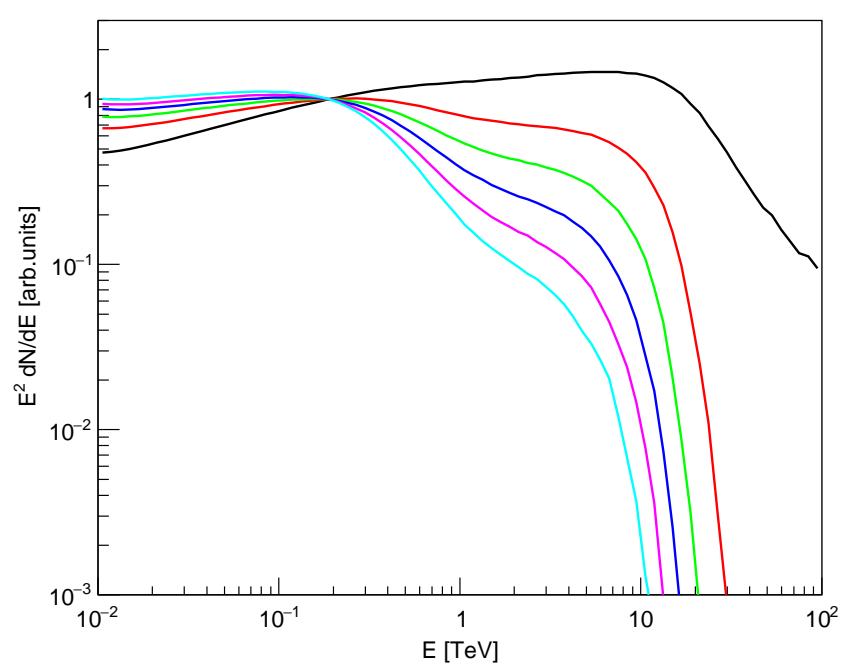

Figure 3. SEDs of intergalactic EM cascades for different intervals of $z_{0}$. Black curve denotes the interval $0.0<z_{0}<0.03$, red $-0.03<z_{0}<$ 0.06 , green $-0.06<z_{0}<0.09$, blue $-0.09<z_{0}<0.12$, magenta $0.12<z_{0}<0.15$, cyan $-0.15<z_{0}<0.18$. For convenience, all curves are normalized to unity at $E=200 \mathrm{GeV}$.

(2013); Stecker et al. (2016); Franceschini \& Rodighiero (2017, 2018). The KD10 option represents a lower limit for the EBL density; G12 is a realistic EBL model that is consistent with existing constraints (Ackermann et al. 2012; Biteau \& Williams 2015; Abdalla et al. 2017; Korochkin \& Rubtsov 2018; Abdollahi et al. 2018; Acciari et al. 2019a; Abeysekara et al. 2019), at least for $z<0.2$.

Qualitatively, the evolution of a well-developed intergalactic EM cascade (i.e. when the parent $\gamma$-ray or electron has a high enough energy $E_{0}>100 \mathrm{TeV}$ for $z_{0}=0.2$ or even higher for lower values of $z_{0}$, usually corresponding to several or more generations of cascade $\gamma$-rays and electrons) may be described as follows (Berezinsky et al. 2011): 1) the parent $\gamma$-ray is absorbed on a EBL or CMB photon, 2) then, the cascade develops very fast, 3) finally, cascade $\gamma$-rays reach the effective $\gamma \gamma$ absorption threshold on EBL $E(\tau=1)$. The observable spectrum carries an imprint of the pair production process, namely, the redshift-dependent highenergy cutoff, that is clearly visible in Fig. 3 for all cases considered. The more distant the cascade origin is, the lower is the cutoff energy in the observable spectrum.

For the primary proton energy in excess of several EeV and below $100 \mathrm{EeV}$, parent electrons and $\gamma$-rays that initiate intergalac- 
tic cascades are mostly produced on CMB photons. In this case, for $E_{p}<60 /(1+z) \mathrm{EeV}$ the dominant energy loss process is the Bethe-Heitler pair production (BHPP), while for $60 /(1+z) \mathrm{EeV}$ $<E_{p}<100 /(1+z) \mathrm{EeV}$ the photohadronic processes rapidly take over (Berezinsky et al. 2006). Furthermore, the typical energy range for photohadronic electrons and $\gamma$-rays is $1-50 \mathrm{EeV}$, while for BHPP electrons it is $100 \mathrm{TeV}-1 \mathrm{EeV}$ (see Figs. 23 in D17). For the range of parameters considered in the present work, the spectrum of observable $\gamma$-rays is practically independent of the energy and the type ( $\gamma$-ray/electron) of the parent particle, but depends on $z_{0}$ ("weak universality", see Berezinsky \& Kalashev (2016) and D17 (Appendix A)). For a discussion of the applicability of the weak universality assumption, the reader is referred to Dzhatdoev et al. (2017); see also Subsect. 6.6.1 and Subsect. 6.6.2 of the present paper.

\subsection{Discussion of estimates}

Above we have shown that the observable angular distribution broadens as the primary proton travels through the EGMF and, at the same time, the observable spectrum becomes harder for the case of cascades that were initiated relatively near to the observer (i.e. for the cascades characterised by comparatively low values of $z_{0}$ ). It is remarkable that the hard component of observable $\gamma$-rays with low $z_{0}$ at the same time has much broader angular distribution than the rest of observable $\gamma$-rays (see values in Table 1 and Fig. 3). This leads to a qualititive conclusion that the spectrum inside the PSF of the observing instrument appears to have a cutoff with respect to the spectrum integrated over all values of the observable angle $\theta_{o b s}$. In the next four Sections we present a more precise calculation of this effect and discuss it in more details.

\section{SIMULATIONS}

\subsection{Deflection of protons}

We simulate the propagation of protons through the EGMF with the publicly available CRPropa3 code (Cosmic Ray Propagation Framework version 3) (Alves Batista et al. 2016), accounting for all relevant particle interactions and calculating energy losses of these protons on every step, assuming the D05 EGMF model. The EGMF is represented by a $(132 M p c)^{3}$ volume simulated using the Quimby code (Müller 2016) and stored in an input file. Reflective boundary conditions were chosen; the trajectories were followed until they reach the observer's sphere. As a result of the proton propagation simulation, we obtained $10^{5}$ trajectories of protons with the primary energy $E_{p 0}=30 \mathrm{EeV}$ and starting points distributed randomly and uniformly inside a $(30 \mathrm{Mpc})^{3}$ cube located in the central region of the volume. The length of each trajectory is $10^{3} \mathrm{Mpc}^{9}$. We conservatively consider the isotropic angular distribution of the protons. ${ }^{10}$

\footnotetext{
9 CRPropa3 allows to propagate particles to greater distances than the extension of the EGMF volume using the replication of the volume

10 for strongly anisotropic angular patterns of the primary proton beam, an additional defocusing effect is in place, which makes the observable spectrum even steeper
}

\subsection{Observable $\gamma$-ray signal}

Following D17, we utilize a hybrid approach and combine semianalytic calculations of proton energy losses during their propagation with statistical (Monte Carlo, MC) simulations of proton deflections (see the previous subsection), as well as MC calculations of intergalactic EM cascade spectra. Coordinates, directions and energies of the protons were calculated with the step $\delta z=10^{-5}$. We accounted for pair production and pion production energy losses on CMB photons, as well as adiabatic losses (redshift) according to Berezinsky et al. (2006) (see Subsection 2.2 of D17). As an additional option we also included pion production energy losses on EBL photons; however, their impact is negligible for the range of parameters considered in the present work. We neglect intra-cluster and intra-filament light (Ellien et al. 2019).

The shape of the observable spectrum of $\gamma$-rays in the weak universality approximation is (D17, eq. 7):

$$
\left(\frac{d N}{d E}\right)_{\gamma-o b s}(E) \propto \int_{0}^{z_{s}} K_{e m}(z) \frac{w(z)}{w(0)}\left(\frac{d N}{d E}\right)_{c}(E, z) d z,
$$

where $K_{e m}(z)$ is the fraction of "active" energy losses (i.e. pair production losses on the $\mathrm{CMB}$, as well as pion production losses on the $\mathrm{CMB}$ and EBL) transferred to $\gamma$-rays and electrons, $\left(d N / d E_{c}\right)(E, z)$ is the universal spectrum of EM cascade with a starting point at $z$, and $w(z) / w(0)$ is the energy transferred to $\gamma$-rays and electrons on the step $d z$, normalized to the value of $w$ at $z=0$. Following Berezinsky et al. (2006):

$$
\begin{gathered}
w(z)=\left|\left(\frac{d E_{p}}{d z}\right)_{\text {pair+pion }}\right|=\left|\left(\frac{d E_{p}}{d t}\right)_{\text {pair }+ \text { pion }}\right|\left|\frac{d t}{d z}\right|, \\
\left|\frac{d t}{d z}\right|=\frac{1}{H_{0} \sqrt{\Omega_{m}(1+z)^{3}+\Omega_{\Lambda}}} \frac{1}{1+z} .
\end{gathered}
$$

Using the ELMAG 2.03 code for the G12 EBL option and the 2.02 version of the same code for the KD10 EBL option (Kachelrieß et al. 2012), we computed a database of the universal EM cascade spectra $\left(d N / d E_{c}\right)(E, z)$ with the primary energy $1 \mathrm{PeV}$ and $z$ distributed randomly and uniformly varying from 0 to 0.30 and use this database to compute the observable spectrum according to eq. (10). Other up-to-date publicly-available intergalactic cascade codes have more advanced functionality than ELMAG 2.03: namely, Kalashev \& Kido (2015) enables the account of the universal radio background (URB), and Fitoussi et al. (2017), as well as the new version of ELMAG (3.01) (Blytt et al. 2019) allow to perform realistic simulation of three-dimensional (3D) structure of intergalactic EM cascades. However, in the scope of the present paper the ELMAG 2.03 code is suitable for our purposes (which are mainly to calculate the energy spectra of intergalactic EM cascades), at the same time retaining significant advantages of greater simplicity, reliability, and speed compared to the more advanced codes.

In our simulation framework the observer is represented by a sphere (denoted as $C$ in Fig. 1 and Fig. 2) with the source in its center, the comoving distance from the source to the observer being the radius of the sphere. Observable $\gamma$-rays are collected at the intersection with the sphere. Thus, the spherical symmetry of our setup allows to greatly simplify the calculations for the case of the isotropic angular distribution of protons.

Finally, we compute and tabulate the observable angle $\theta_{o b s}$ for every proton trajectory simulated as was described in Subsect. 3.1. These tables have the step of $5 \mathrm{Mpc}$ on the traversed distance; in 
what follows we use them to calculate the observable $\gamma$-ray angular distribution.

\section{RESULTS}

\subsection{Observable spectrum with and without the account of proton deflection}

In order to illustrate the original idea of Uryson $(1998)^{11}$, in this Section we present results with and without the account of primary proton deflection and compare them.

In D17 we called the version of the IHCM neglecting proton deflection "the basic intergalactic hadronic cascade model" (BHCM) and calculated the observable spectra for a set of sources using this model. Now we repeat the calculation of the observable spectrum, taking the observable angle into consideration. We retain only those observable $\gamma$-rays that have $\theta_{o b s}<\theta_{P S F}=0.1^{\circ}$ and reject those with $\theta_{o b s} \geq \theta_{P S F}$. Results for a more realistic PSF are presented below in Subsect 4.2. In D17 we had already shown that the effect of proton deflection strongly modifies the observable spectrum, therefore, we called this version of the IHCM "the modified intergalactic hadronic cascade model" (MHCM).

Here we present, for the first time, the results of detailed calculations based on a model of LSS formation predicting a highly inhomogeneous EGMF, namely, the D05 model. They are presented in Fig. 4 in the form of an observable SED (blue curve). The SEDs for the BHCM (black curve) and the elecromagnetic cascade model $(E C M)$ in the universal regime (red curve) are presented as well; all three curves were calculated assuming the KD10 EBL option ${ }^{12}$.

As one can see from Fig. 4, the observable spectrum in the framework of the BHCM is much harder than the universal EM cascade spectrum. As was explained in the Introduction, the reason behind this effect is that a part of EM cascades initiated by the protons have the redshift of the origin $z_{0} \ll z_{s}$, and so these cascades have much harder spectra than those with $z_{0}=z_{s}$ (see also Fig. 3 and the related discussion). To put it simply, in the framework of the BHCM the source becomes effectively much nearer to the observer, reducing the impact of intergalactic $\gamma \gamma$ absorption on the observable spectrum.

After the account of proton deflection, however, the situation appears to be very different with respect to the oversimplified case of the BHCM. We note that there is a marked difference between the shapes of the corresponding SEDs in the framework of the basic and modified hadronic models. The MHCM SEDs have a highenergy cutoff at $E \sim 10 \mathrm{TeV}$. This cutoff is due to the relatively broad angular distribution of observable $\gamma$-rays from cascades initiated near the observer. The results presented in Fig. 4 very well match our expectations based on the estimates made in Sect. 2.

In Fig. 5 we present the results of a calculation similar to the one presented in Fig. 4, but done assuming the G12 EBL option. Observable SEDs for the case of the MHCM and the KD10 EBL model option were calculated by averaging over $10^{4}$ proton trajectories and $10^{3}$ proton trajectories were averaged for the G12 EBL option. We have found that averaging over $10^{3}$ trajectories

11 many works, including Essey et al. (2010, 2011); Zheng et al. (2015); Archer et al. (2018) are basically founded on the same idea as the one proposed by Uryson (1998)

12 the SED of the BHCM presented in Fig. 4 was normalized to unity at its maximal value; the SED for the ECM in the universal regime was normalized to the SED of the MHCM at $E=100 \mathrm{MeV}$

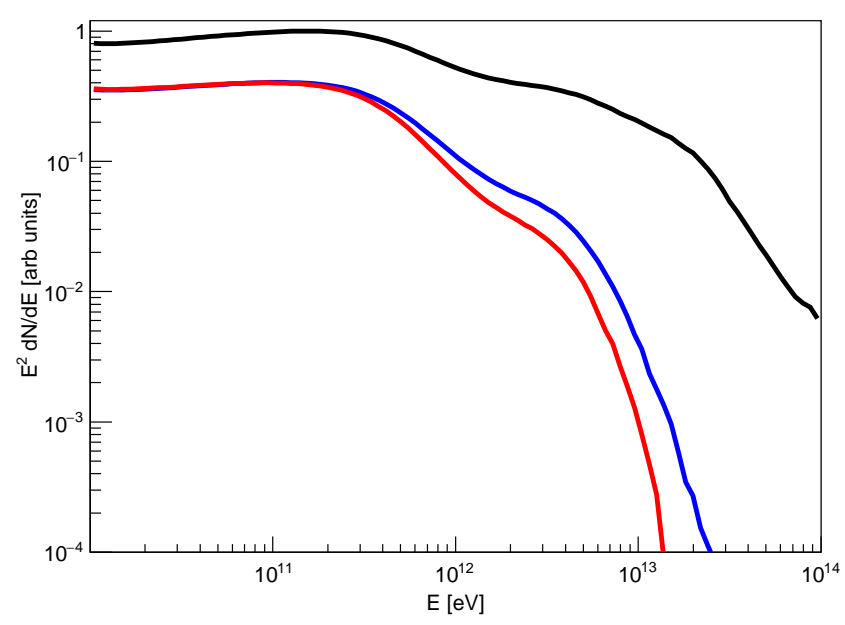

Figure 4. Observable SEDs for intergalactic cascade models for $z_{s}=0.186$ and the KD10 EBL option. Black curve denotes an SED for the basic hadronic cascade model, blue curve — an SED for the modified hadronic cascade model. Red curve denotes an SED for the intergalactic electromagnetic cascade model ("the universal SED").

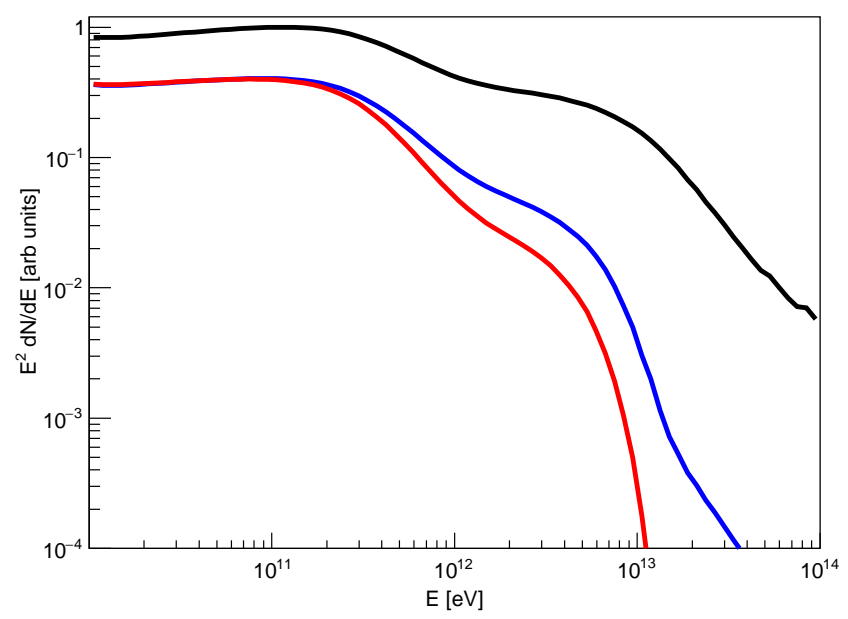

Figure 5. The same as in Fig. 4, but for the G12 EBL option.

is sufficient to obtain stable enough results for $E<30 \mathrm{TeV}$ (see Appendix A, Fig. A1 for details). Observable $\gamma$-ray spectra for $10^{2}$ individual trajectories are also presented in Appendix A (see Fig. A2). We also note that the difference between the spectra of the modified hadronic models and the universal spectra of purely EM cascades is comparable to the difference between these universal spectra for various EBL options.

It is useful to introduce a new parameter called "the critical distance" $L_{c r}$ defined as the distance from the observer at which the observable angle is equal to the typical extension of the PSF (e.g. the $68 \%$ containment angle): $\theta_{o b s}=\theta_{P S F}$. A histogram of the distribution on $L_{c r}$ for $\theta_{P S F}=0.1^{\circ}$ is shown in Appendix B (see Fig. B1) ${ }^{13}$. This histogram is peaked at $L_{c r} \approx 200 \mathrm{Mpc}$ with only a small minority of trajectories having $L_{c r}>300 \mathrm{Mpc}$.

The relative contributions of extragalactic filaments and clus-

13 here $L_{c r}$ was calculated for individual trajectories 


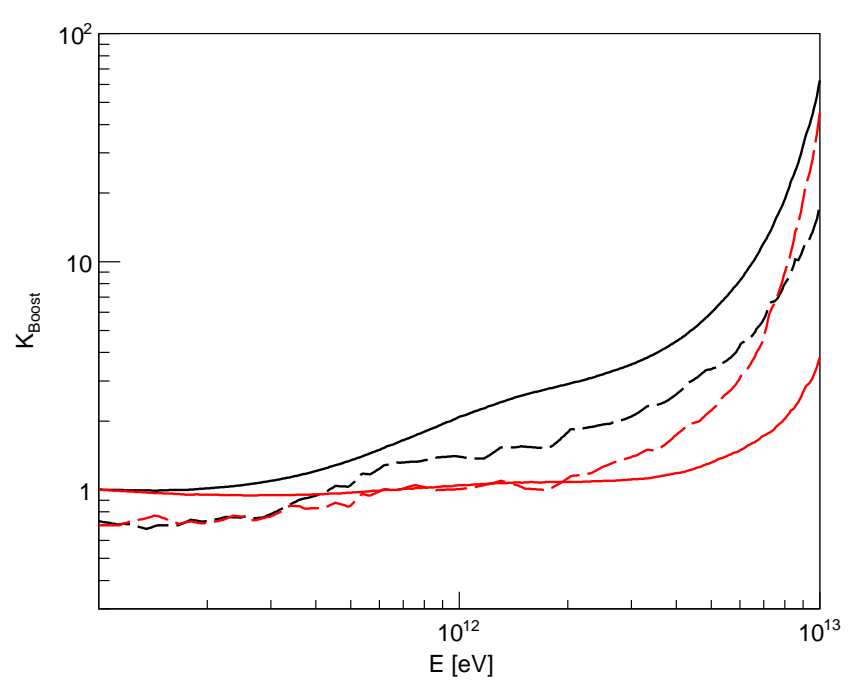

Figure 6. Flux boost factors for the BHCM (black solid curve), MHCM (red solid curve), and two models including the $\gamma \rightarrow A L P$ oscillation effect with $\xi=5.0$ (black dashed curve) and $\xi=0.5$ (black dashed curve).

ters to the total proton deflection vary with the redshift of the source. A border between the clusters and the filaments on the plane of the variables "deflection angle - the cumulative fraction of the sky with deflection angle larger than some threshold" is shown in Fig. 15 of D05 as dot-dashed magenta line. We estimate that for $z=0.186$ the fraction of the sky covered by clusters is $\approx 0.2$. For $z=0.3-0.4$ this fraction rises to 0.5 ; therefore, for larger $z$ the proton deflection is typically dominated by galaxy clusters. Additionally, the typical deflection in a cluster is significantly larger than the one in a filament (Murase et al. 2012). We note that the results of such estimates are strongly dependent on a particular line-of-sight chosen.

Finally, we calculated the so-called flux boost factors $K_{\text {Boost }}(E)$ (Sánchez-Conde et al. 2009), defined as the ratio between the spectrum in the framework of a certain model to the spectrum in the framework of the absorption-only model. For BHCM and MHCM, the "absorption-only" spectrum was assumed according to Fig. 12 of Dzhatdoev et al. (2017) (top-left) and the G12 EBL model (denoted as black curve in this figure). Additionally, we calculated $K_{\text {Boost }}(E)$ for two models including the $\gamma \rightarrow A L P$ oscillation effect according to De Angelis et al. (2011), Fig. 3 (second row, left) that were developed by the authors of this paper for $z=0.188$, the EGMF coherence length of $4 \mathrm{Mpc}$, and two values of $\xi \equiv\left(B_{0}[n G]\right)\left(10^{11}[\mathrm{GeV}] / M\right)$ (here $M=1 / g$ and $g$ is the ALP-photon coupling constant; De Angelis et al. (2011) assumed the EGMF strength evolution $\left.B=B_{0}(1+z)^{2}\right) \cdot{ }^{14}$ Flux boost factors in the energy range from $100 \mathrm{GeV}$ to $10 \mathrm{TeV}$ for the four abovementioned models are shown in Fig. 6.

For the basic hadronic model the modification factor is comparable with those for both of $\gamma \rightarrow A L P$ models. Therefore, in the framework of the BHCM, intergalactic cascades initiated by the primary protons indeed represent a dangerous source of background in ALP searches. For the MHCM, however, the modifica-

14 De Angelis et al. (2011) assumed the EBL model of Franceschini et al. (2008) that for $z<0.2$ predicts the EBL parameters close to those predicted by the G12 model

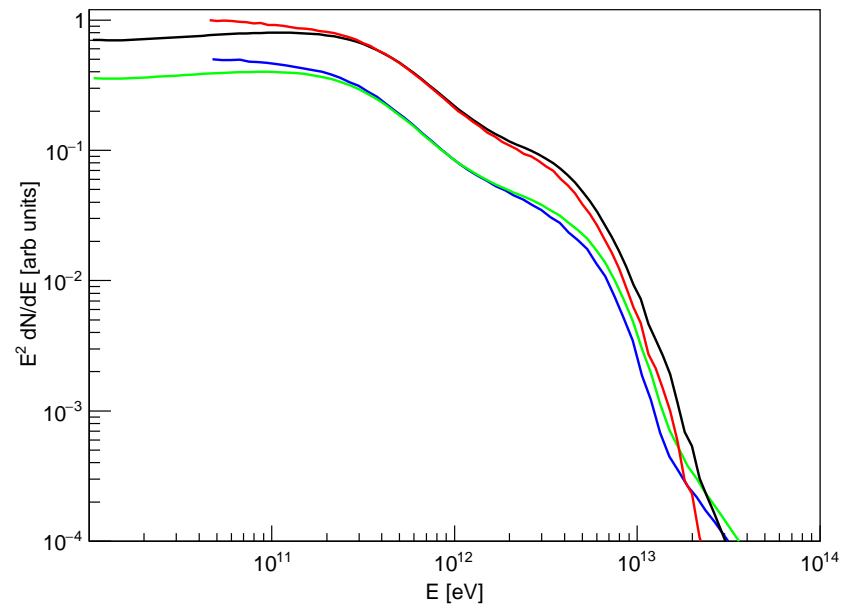

Figure 7. A comparison between the SEDs for a simplified PSF with $\theta_{P S F}=0.1^{\circ}$ and a realistic one (for the H.E.S.S. array) in the MHCM. Black curve denotes an SED for a simplified PSF and the KD10 EBL option, green curve - the same for the G12 EBL option. Red curve denotes an SED for the H.E.S.S. PSF and the KD10 EBL option, blue curve - the same for the G12 EBL option. SEDs with different EBL options are spaced apart by the factor of two for illustrative purposes.

tion factor at $E>3 \mathrm{TeV}$ is significantly smaller than for both of $\gamma \rightarrow A L P$ models, allowing to distinguish between these models.

\subsection{Observable spectrum: the case of a realistic PSF}

The width of the PSF for most of the contemporary $\gamma$-ray detectors depends on the energy. This impacts the shape of the observable SED in the framework of the MHCM. In Fig. 7 we compare SEDs for a simplified representation of a PSF with $\theta_{P S F}=0.1^{\circ}$ and a more realistic PSF for the case of the H.E.S.S. IACT array (The CTA Consortium 2018). The SEDs for both cases were averaged over $10^{4}$ trajectories for the KD10 EBL option and over $10^{3}$ trajectories for the G12 option.

The H.E.S.S. PSF becomes wider at lower energies, so the intensity at low energies appears to be greater than the one for the case of $\theta_{P S F}=0.1^{\circ}$. Thus, for the realistic PSF the observable spectrum becomes steeper than for the case of an energy-independent PSF.

\subsection{Observable spectrum: comparison with observations}

In Fig. 8 we show an SED of the extreme $\mathrm{TeV}$ blazar 1ES 1101-232 $\left(z_{s}=0.186\right)$ measured by the H.E.S.S. Collaboration (Aharonian et al. 2006). In 2007 the H.E.S.S. Collaboration had performed a reanalysis of the same dataset (Aharonian et al. 2007a); the resulting SED is also presented in Fig. 8 for comparison. In the same figure we show two model curves in the framework of the MHCM for various EBL models; the realistic PSF of the H.E.S.S. IACT array was assumed for both model options. ${ }^{15}$

In Fig. 9 we show an SED of the extreme TeV blazar 1ES $0347-121\left(z_{s}=0.188\right)$ that was also measured by the H.E.S.S. Collaboration (Aharonian et al. 2007b), and present the same two

15 these model curves were shown in Fig. 7 (see Subsect. 4.2 for more details); here we renormalized them to fit the measured SEDs 


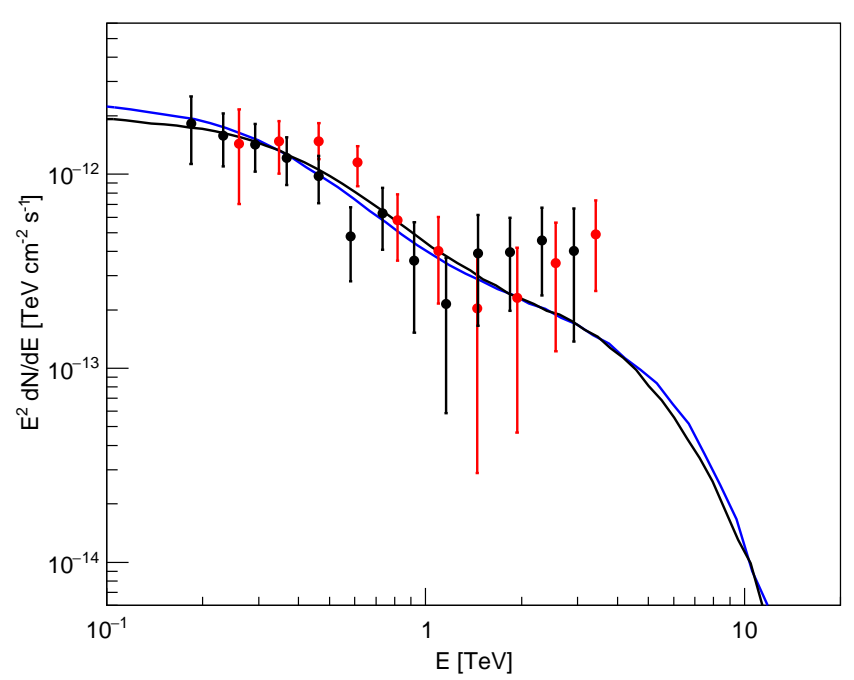

Figure 8. SEDs of the source 1ES1101-232 measured with the H.E.S.S. IACT array: circles denote measurements, bars - their uncertainties; the results of the 2006 analysis are shown in black, the results of the 2007 reanalysis - in red. Black curve denotes the SED for the MHCM assuming the H.E.S.S. PSF and the KD10 EBL option, blue curve — the same, but for the G12 EBL option.

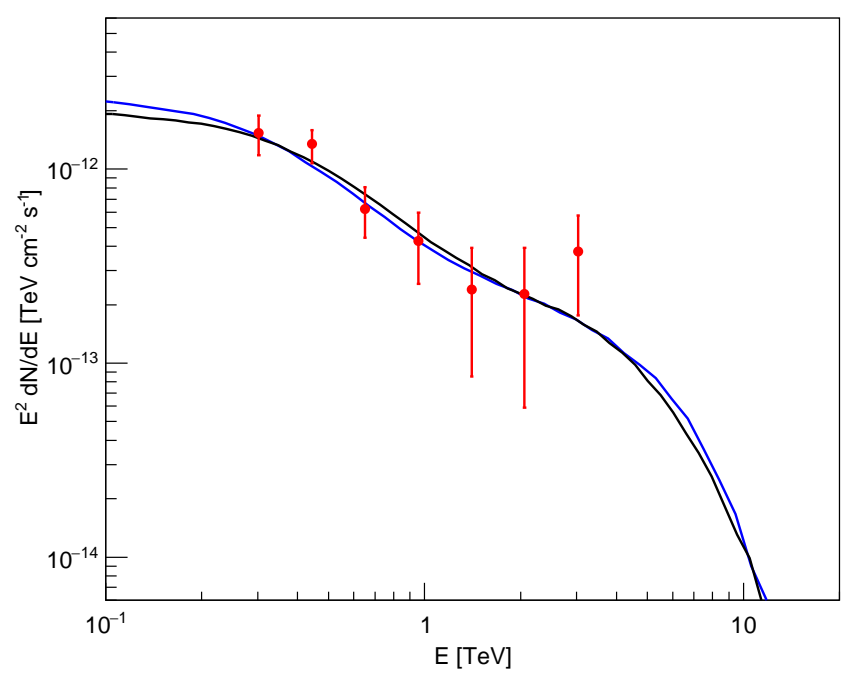

Figure 9. The same as in Fig. 8, but the measurements are shown for the source 1ES 0347-121.

model curves as in Fig. 8. ${ }^{16}$ For both blazars, the figures demonstrate a reasonable agreement between the observed SEDs and the model ones, except for a slight excess in the measured SEDs at the highest energies.

\subsection{Observable angular distribution}

Besides the energy spectrum, $\gamma$-ray detectors can also measure the angular distribution of the source. In particular, the Fermi-LAT

16 the small difference of the measured redshift between 1ES 1101-232 and 1ES 0347-121 allows one to use the same models

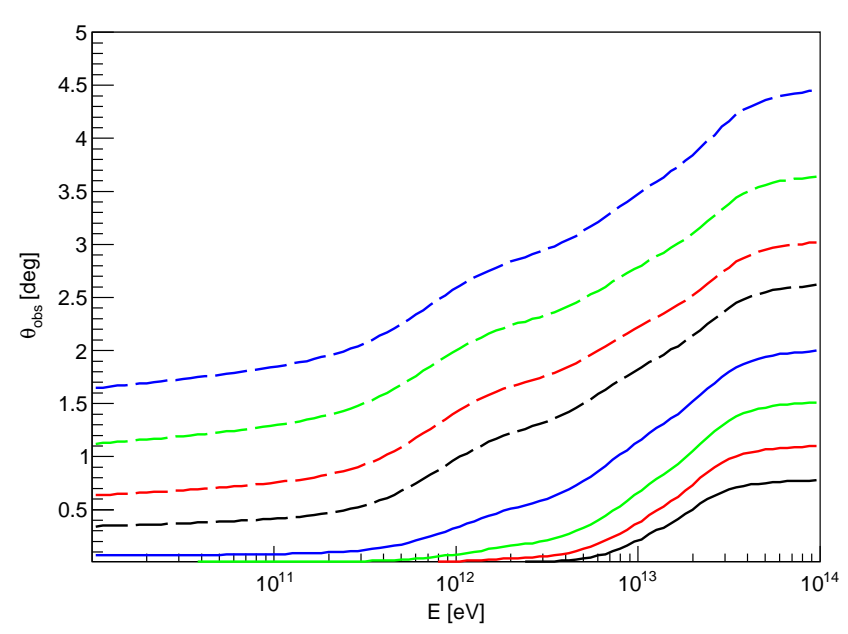

Figure 10. Quantiles of the observable angular distribution for the KD10 EBL model option: black solid curve denotes a 5\% containment angle, red solid curve $-10 \%$ containment angle, green solid curve $-20 \%$, blue solid curve $-40 \%$, black dashed curve $-68 \%$, red dashed curve $-80 \%$, green dashed curve $-90 \%$, and blue dashed curve $-95 \%$.

Collaboration have recently performed a search for spatial extension in some high-latitude $\left(|b|>5^{\circ}\right)$ sources, including the blazars 1ES 1101-232 and 1ES 0347-121 (Ackermann et al. 2018b). They have drawn the conclusion that the images of these sources are compatible with the ones expected from point-like sources. The H.E.S.S. Collaboration have presented the same conclusion in Aharonian et al. (2007b) for 1ES 0347-121; the observable angular distribution for 1ES 1101-232 also does not show any obvious hints at an additional extension (Aharonian et al. 2007a).

In Fig. 10 we show a model of the observable angular distribution that could be compared with observations; this figure demonstrates how various containment values of the observable angle $\theta_{\text {obs }}$ change with the energy. ${ }^{17}$ We note that there is a steady increase in the value of $\theta_{\text {obs }}$ as the observable energy increases, with $\approx 32 \%$ of observable angles being larger than $1^{\circ}$ at $E>1 \mathrm{TeV}$, and $\approx 60 \%$ of observable angles exceeding this value at $E>8 \mathrm{TeV}$.

Thus, the intergalactic hadronic cascade model for the blazars 1ES 1101-232 and 1ES 0347-121 predicts observable spectra similar to those measured by the H.E.S.S. Collaboration, but this model is not favoured in view of observed angular distributions typical for point-like sources. A detailed statistical analysis supporting this conclusion is in preparation; it will be published elsewhere. Here we conclude that measurement of the observable angular distribution would provide a critical test for the validity of the intergalactic hadronic cascade model.

\section{OBSERVATIONAL PROSPECTS}

In this section we compare the $68 \%$ containment radius of the simulated angular distribution with the same quantity for the PSF of several operating and projected $\gamma$-ray telescopes (see Fig. 11). In this figure we present the single-photon angular resolution $\theta_{68}(E)$ for Fermi-LAT according to Atwood et al. (2009); $\theta_{68}(E)$ for H.E.S.S.,

17 here we work with the angular distribution truncated at $10^{\circ}$; this value represents the diameter of the field-of-view of a typical IACT or a typical extension of the region-of-interest (ROI) in Fermi-LAT data analysis 


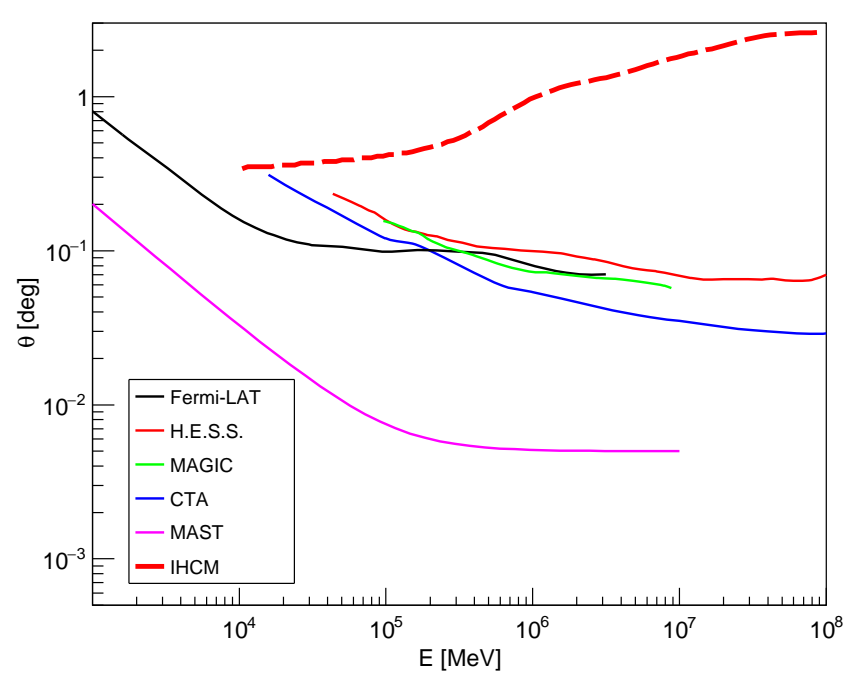

Figure 11. Angular resolution (68\% containment angle) of various gammaray instruments (solid curves: black curve denotes Fermi-LAT, red curve H.E.S.S., green curve - MAGIC, blue curve - CTA, magenta curve $M A S T)$ vs. the $68 \%$ containment angle of the observable emission assuming the MHCM with the KD10 EBL.

MAGIC and CTA are taken from (The CTA Consortium 2018). The angular resolution for VERITAS (Krennrich et al. 2004; Park 2016) is similar to that of H.E.S.S. and MAGIC. In addition, we show the angular resolution for the MAST space $\gamma$-ray telescope proposed in Dzhatdoev \& Podlesnyi (2019). The MAST concept (an abbreviation for "Massive Argon Space Telescope") is based on the liquid Argon time projection chamber (TPC) approach (Dolgoshein et al. 1970; Rubbia 1977; Rubbia et al. 2011). Such a detector with the total sensitive mass of $\approx 36 \mathrm{t}$ could be launched with the latest contemporary launch vehicles such as Falcon Heavy.

Finally, in Fig. 11 we also present the $68 \%$ containment angle of the observable emission in the framework of the MHCM. We note that this extended emission is readily discernible by all considered $\gamma$-ray telescopes at $E>10 \mathrm{GeV}$ provided that the exposure is sufficiently high and the background is sufficiently low. Nextgeneration IACTs such as CTA are especially well-suited for the search of broadened patterns around the positions of presumably point-like sources as the extension of the model angular distribution significantly increases with the energy. Projected space $\gamma$-ray telescopes with improved angular resolution, broad field-of-view, and high duty cycle, such as MAST, could also reveal the extended nature of these sources.

In Fig. 12 we show the SEDs of extreme TeV blazars 1ES 1101-232, 1ES 0347-121, and 1ES 0229+200 $\left(z_{s}=0.14\right)$ measured with H.E.S.S. (Aharonian et al. 2007c,b), VERITAS (Aliu et al. 2014), and Fermi-LAT (Ackermann et al. 2018b) in comparison with the differential sensitivity for point-like sources of various $\gamma$-ray telescopes. The sensitivity for MAGIC and VERITAS is qualitatively similar to that of H.E.S.S. The sensitivity of Fermi-LAT is barely sufficient to measure the spectra of these sources; however, IACTs can readily detect extreme TeV blazars due to their very hard intrinsic spectra. Next-generation space $\gamma$ ray telescopes such as MAST could also measure the spectra of such sources due to improved sensitivity.

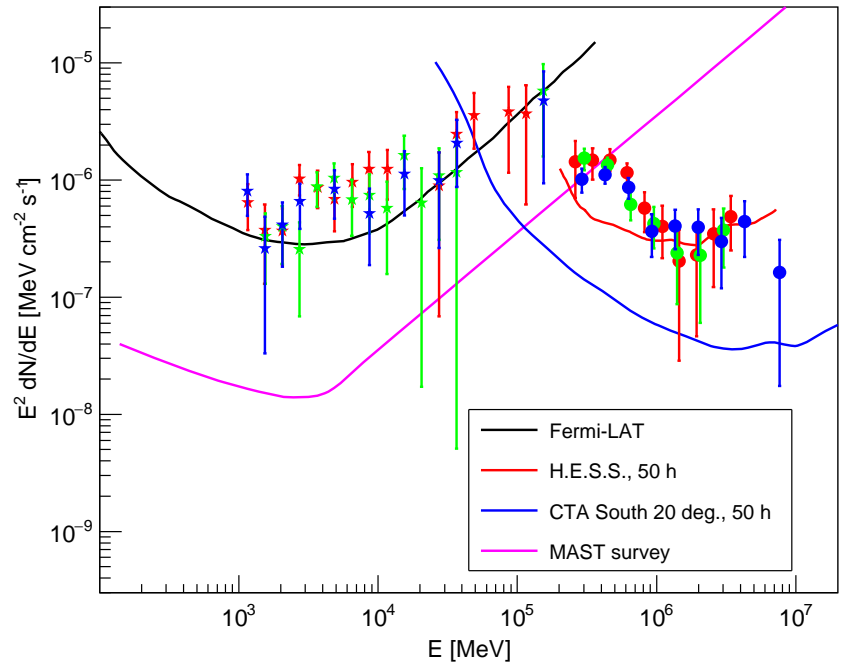

Figure 12. SEDs of some extreme TeV blazars measured with IACTs (circles) and Fermi-LAT (stars) together with sensitivities of several $\gamma$ ray telescopes. Red symbols denote 1ES 1101-232, green symbols 1ES 0347-121, blue symbols - 1ES 0229+200; black curve denotes the sensitivity of Fermi-LAT, red curve - H.E.S.S., blue curve - CTA, magenta curve - MAST.

\section{DISCUSSION}

For the calculations performed in the present paper we assumed specific model parameters, including the source redshift $z_{s}=0.186$, the primary proton energy $E_{p 0}=30 \mathrm{EeV}$, the D05 model for the EGMF, and two models of the EBL (namely, KD10 and G12). Here we discuss how the results of our calculations would change if we assume other values of these parameters or account for "additional processes", such as triplet pair production (TPP, $e \gamma \rightarrow 3 e$ ), double pair production (DPP, $\gamma \gamma \rightarrow 4 e$ ), and interactions on the URB.

\subsection{Source redshift}

At redshifts higher than $z_{s}=0.3$ the fraction of proton trajectories that experience deflections on the EGMF of galaxy clusters becomes non-negligible, significantly increasing the typical deflection angle and making the effect of the angular broadening and the associated cutoff in the observable spectrum even more pronounced. Conversely, at relatively low redshifts $\left(z_{s}<0.1\right)$ a significant deviation of the observable spectrum in the framework of the MHCM from that of the universal spectrum of a purely EM cascade is expected. On the other hand, the width of the observable angular distribution rises with increasing energy, while the angular resolution of IACTs becomes better with the energy (see Fig. 10 and Fig. 11), making the angular extensions of the sources readily discernible, even for the case of low redshifts.

\subsection{Primary proton energy}

For the case of the primary proton energy lower than $30 \mathrm{EeV}$, the primary protons deflect even stronger and the width of the observable angular distribution increases still further. The same is true for primary nuclei with the primary energy $<30 \cdot \mathrm{Z} \mathrm{EeV}$. We note that the results obtained with the Pierre Auger Observatory disfavour the hypothesis of a purely proton composition above the energy of $10 \mathrm{EeV}$ (Abraham et al. 2010; Aab et al. 2014, 2017a). 
For the case of high $E_{p 0}>100 \mathrm{EeV}$, the characteristic energy loss length $L_{p-E}$ is lower than $100 \mathrm{Mpc}$ (Berezinsky et al. 2006). Therefore, at first, protons lose energy rapidly until they reach the effective threshold of the pion photoproduction process $(\sim 60 /(1+$ z) EeV). Because of this, the width of the angular distribution at high energies $(E>1 \mathrm{TeV})$ becomes only slightly smaller for $E_{p 0} \gg$ $30 \mathrm{EeV}$ as compared to the case of $E_{p 0}=30 \mathrm{EeV}$ if the source is sufficiently distant, $z_{s}>0.1$.

\subsection{EGMF model}

The D05 EGMF model is usually considered to be conservative in terms of the predicted proton deflection, i.e. it predicts proton deflection to be smaller than the predictions of other models such as Sigl et al. (2004); Hackstein et al. (2018) (see Eichmann (2019), Subsect. 2.2). An attempt to reduce the angular extension of the observable emission beyond the observability level for contemporary experiments such as the $C T A$ array would require a significant revision of the existing models of the EGMF in filaments and clusters of the large scale structure.

First detections of synchrotron emission from extragalactic filaments have just started to appear (Govoni et al. 2019; Vacca et al. 2018). Based on the LOFAR observations reported in Govoni et al. (2019), Brunetti \& Vazza (2020) derived $B=500-600 \mathrm{nG}$ for the filament connecting the galaxy clusters Abell 0399 and Abell 0401, much in excess of the typical $B$ in the framework of the D05 model. It is likely that the filaments detected in Govoni et al. (2019); Vacca et al. (2018) represent only a "tip of the iceberg" of the more numerous population of extragalactic filaments with a weaker magnetic field but a higher total volume filling factor. Future observations with more sensitive instruments, such as the SKA array (Heald et al. 2020), would likely reveal these fainter filaments. Concerning the assumptions and uncertainties of EGMF simulations, the reader is referred to Sigl et al. (2004); Dolag et al. (2005); Vazza et al. (2017); Hackstein et al. (2018); Alves Batista et al. (2019). Results assuming a more up-to-date EGMF model compared to D05 are in preparation and will be published elsewhere.

The ratio of the synchrotron and Compton luminosities of electrons in a filament is $P_{S} / P_{I C} \sim\left(B / B_{c m b}\right)^{2}$ (Brunetti \& Vazza 2020), where $B_{c m b}=3.25 \mu \mathrm{G} \cdot(1+z)^{2} .{ }^{18}$ Therefore, detecting intergalactic filaments by observing their synchrotron radiation is difficult because for $B<1 \mu \mathrm{G}$ most of the radiated power is transferred to Compton photons rather than to synchrotron ones. This could explain the small number of intergalactic filaments with synchrotron radiation detected so far, which results in the current low number of EGMF measurements in these filaments. Observations with X-ray missions such as XMM-Newton (Jansen et al. 2001), Chandra (Wilkes \& Tucker 2019), SWIFT (Gehrels et al. 2004), $S R G$ (Merloni et al. 2012) as well as with the proposed Athena Xray observatory (Nandra et al. 2013) could provide additional constraints on the value of $B$ or even result in the measurement of the EGMF strength in some extragalactic filaments.

\subsection{EBL model}

The intensity of the EBL relevant for primary $\gamma$-ray absorption in the observable energy range $E<20 \mathrm{TeV}$ is believed to be

18 indeed, the number of Compton scattering acts is $\propto(1+z)^{3}$, and the average energy of produced $\gamma$-rays is $\propto(1+z)$

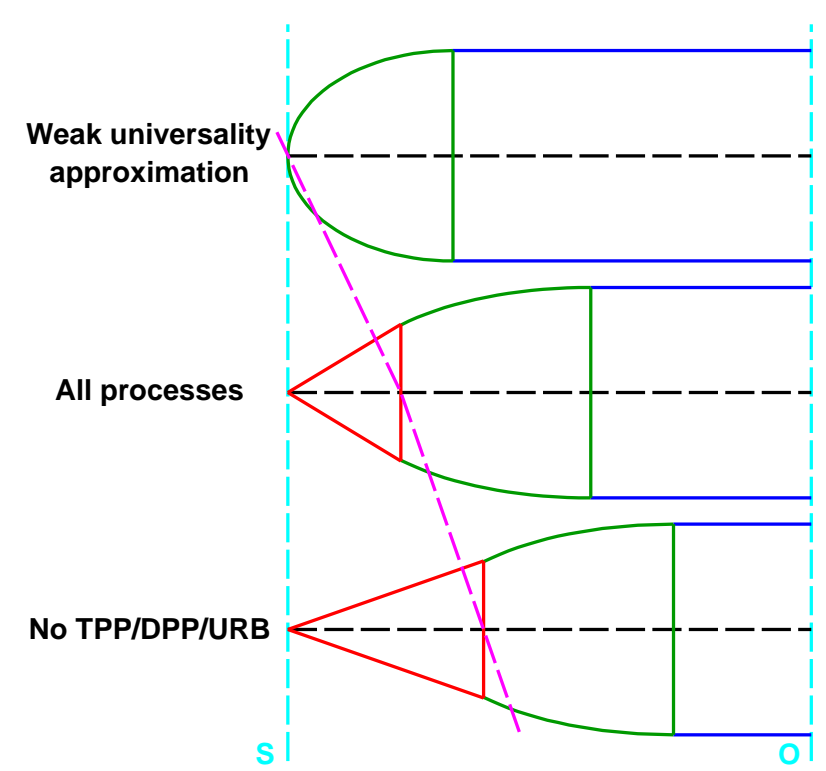

Figure 13. Simplified scheme of geometry of electromagnetic cascade. Three main development stages of the cascade are shown: the high energy stage, the particle multiplication stage, and the low energy stage. Red cones denote the high energy stage, green parabolic segments — the particle multiplication stage, blue parallel lines - the low energy stage. Top panel denotes the weak universality approximation, middle panel - the case when all processes are taken into consideration, bottom panel - the case when the additional processes are neglected. $\mathrm{S}$ denotes the source, $\mathrm{O}$ - the observer. To the right of the dashed magenta line the additional processes could be safely neglected. We note that the length of the high energy stage is lower when all processes are accounted for compared to the case when the additional processes are neglected (see the text for more details).

well-constrained now (Ackermann et al. 2012; Abdalla et al. 2017; Abdollahi et al. 2018; Acciari et al. 2019a; Abeysekara et al. 2019; Franceschini et al. 2019; Biasuzzi et al. 2019), with characteristic uncertainty of the order of tens of percent at $z<0.5$. In the present paper we have shown the results of calculations assuming the KD10 EBL model that represents a lower limit for the EBL intensity. Other EBL models with higher intensities would result in an additional steepening of the observable spectrum with respect to the KD10 model one.

\subsection{Angular distribution of primary protons}

For simplicity, in the present paper we assumed the isotropic angular distribution of primary protons. Therefore, the results presented here are relevant for the case of $\theta_{p} \gg \delta \sim 1^{\circ}$, where $\theta_{p}$ is the width of the angular distribution of primary protons. For $\theta_{p} \leq \delta$, instead, an additional effect of the beam defocusing is in place, for which the intensity at high energies is diminished further, thus, making the high-energy cutoff in the observable spectrum even more pronounced. 


\subsection{The impact of URB, TPP, and DPP on the observable spectrum}

\subsubsection{Qualitative discussion}

Interactions on the URB as well as the TPP and DPP processes can have an impact on the observable spectrum. URB intensity estimates were obtained in Protheroe \& Biermann (1996); Niţu et al. (2021). The TPP process was considered in Jarp \& Mork (1973); Mastichiadis et al. (1986), the DPP process - in Brown et al. (1973); Lee (1998). Mastichiadis et al. (1994) investigated the consequences of including TPP into calculations of EM cascade development. They reached the general conclusion that "the importance of triplet production decreases as the path length increases" and that TPP "has no effect on saturated cascades", i.e. cascades with $\gamma$-rays below the pair production threshold.

Since the ELMAG code, which we use in this work to calculate cascades, does not account for these additional processes, below we aim to put the results of Mastichiadis et al. (1994) firmly within the context of the present work and justify the use of ELMAG.

Let us consider various stages of EM cascade development (see Fig. 13). The top panel of this figure corresponds to the case of the weak universality approximation. In this case the cascade enters the "particle multiplication stage" immediately, i.e. the energy of particles is divided roughly in equal proportions between the secondaries. This corresponds to the case of the primary $\gamma$-ray/electron energy $E_{0}=1-10 \mathrm{PeV}$. In two other cases, the cascade may first enter the "high energy stage" if the primary energy is high enough $\left(E_{0}>100 \mathrm{PeV}\right)$; in this stage one of the secondaries may carry almost all of the primary energy. Finally, the last stage of cascade development (after the particle multiplication stage) is the "low energy stage"; at this stage the remnant cascade photons gradually become "sterile" (Berezinsky \& Kalashev 2016), i.e. their pair production mean free path becomes much greater than the distance from the source to the observer. These stages were discussed in Berezinsky \& Kalashev (2016).

Dashed magenta line in Fig. 13 denotes the start of the particle multiplication stage. After this stage is set, the additional processes could be safely neglected, because the energy of the cascade particles at this point is typically below $10 \mathrm{PeV}$. We note that the spectral universality is the property of the particle multiplication stage. If the primary energy is high enough, then the cascade first enters the high energy stage, and the universality assumption is, to some extent, violated, even if no additional processes, such as the interactions on the URB as well as the TPP and DPP processes, are accounted for.

The extent to which the universality assumption is violated is defined by the following parameter: the ratio of the typical length of the high energy stage to the distance from the source to the observer. In the next subsection we show that the typical length of the high energy stage is actually lower for the case when the additional processes are accounted for compared to the case when these additional processes are neglected. Therefore, the observable spectra obtained in the "weak universality" approximation are actually less different from the spectra calculated with the account of all relevant processes than the universal spectra compared to the spectra without the account of the additional processes.

\subsubsection{Estimates}

Let us consider the development of intergalactic EM cascades for the case of the primary particle energy $E_{0}>100 \mathrm{PeV}$, at first ne- glecting the interactions on the URB as well as the TPP and DPP processes. In this case the average fraction of the primary energy lost in each collision (usually called "average inelasticity") is (e.g. eq. (4) of Berezinsky \& Kalashev (2016)):

$$
f_{\text {in }} \approx \frac{1}{\ln (2 x)},
$$

where the parameter $x=E_{0} E_{b} /\left(m_{e} c^{2}\right)^{2}$ is typically $\gg 1$ (here $m_{e}$ is the mass of electron and $E_{b}$ is the background photon energy). Therefore, one of the secondaries typically carries almost all of the primary energy, and the cascade is in the high-energy or leadingparticle stage: $\gamma \rightarrow e \rightarrow \gamma^{\prime} \rightarrow e^{\prime}$ (Berezinsky \& Kalashev 2016).

In this paper we mainly consider the case of the primary proton energy $E_{p 0} \leq 30 \mathrm{EeV}$ (see Subsect. 6.2 above). For moderate redshifts $z<0.5$ such protons lose energy mainly through pair production; the typical energy of the produced electrons is below 1 $\mathrm{EeV}$ (see e.g. Fig. 2 of Dzhatdoev et al. (2017)). The mean free path for $\gamma$-rays and electrons with the energy between $100 \mathrm{PeV}$ and $100 \mathrm{EeV}$ could be estimated as:

$$
\lambda_{\gamma, e} \approx C_{\gamma, e}\left(\frac{E_{0}}{100 \mathrm{PeV}}\right)^{0.85},
$$

where for $\gamma$-rays $C_{\gamma}=50 \mathrm{kpc}$, and for electrons $C_{e}=80 \mathrm{kpc}$.

The length of the high energy stage may be estimated as the typical length of the leading-particle chain $L_{\text {lead }} \sim \lambda / f_{\text {in }} ; L_{\text {lead }} \sim$ $300-500 \mathrm{kpc}$ for $E_{0}=100 \mathrm{PeV}$ and $L_{\text {lead }} \sim 3-5 \mathrm{Mpc}$ for $E_{0}=1$ EeV. Beyond the distance of $(2-3) L_{\text {lead }}$ the cascade enters the particle multiplication stage when the energy is quickly (length scale $\sim(2-3) \lambda)$ transferred to many secondaries; the number of these secondaries after the generation $N_{\text {casc }}$ is $2^{N_{\text {casc }}} .{ }^{19}$

For the range of parameters of the present study interactions on the URB as well as the TPP and DPP processes have one common feature: these are mostly near-threshold interactions with the average inelasticity $f_{\text {in }} \sim 0.5$. In this case, the particle multiplication stage sets in much faster than in the case when additional processes are neglected. The effects of the DPP process are qualitatively similar to those of the TPP process, but DPP is relevant at higher energies than TPP.

We illustrate these qualitative considerations and estimates with detailed numerical simulations performed with the CRPropa3 code in Appendix C. In particular, we show that in the case when all three processes under investigation are accounted for, almost all of $\gamma$-rays have the energy below $200 \mathrm{TeV}$ already after the propagated distance of $10 \mathrm{Mpc}$. After this distance, the ELMAG code is fully applicable.

In the present work, it will be remembered, we assume weak universality ((Berezinsky \& Kalashev 2016), see Subsect. 3.2); this assumption is even better justified if the interactions on the URB as well as the TPP and DPP processes are included into calculations. We have also checked that for the propagated distance comparable to the distance from the source to the observer the shape of the observable spectrum is indeed well described by the distantdependent universal spectrum. Finally, we note that a comparison of the observable spectra in the weak universality approximation and in the "full hybrid approach" (without this assumption) is presented in Dzhatdoev et al. (2017). In particular, for the redshift of the source $z>0.02$ the weak universality assumption is valid even if one does not account the interactions on the URB as well as the TPP and DPP processes.

19 during the particle multiplication stage the effect of interactions on the URB as well as the TPP and DPP processes could be safely neglected 


\subsection{Collective (plasma) energy losses}

Cascade electrons may lose energy through the excitation of plasma instabilities (Broderick et al. 2012). The impact of such collective (plasma) energy losses on the observable spectrum is, at present, not well understood (e.g. (Schlickeiser et al. 2012; Miniati \& Elyiv 2013; Chang et al. 2014; Sironi \& Giannios 2014; Menzler \& Schlickeiser 2015; Kempf et al. 2016; Vafin et al. 2018, 2019)). For this reason we did not account the plasma losses in the present work. The inclusion of this additional channel of energy losses would decrease the observable $\gamma$-ray intensity.

\section{CONCLUSIONS}

In this work we calculated the observable spectral and angular distributions of very high energy cascade $\gamma$-rays as expected from extragalactic sources of ultrahigh energy protons. In particular, we accounted for the effect of primary proton deflection in an inhomogeneous extragalactic magnetic field. We demonstrated that the observable spectrum reveals a high-energy cutoff in comparison with the spectrum averaged over all values of the observable angle $\theta_{o b s}$, and that the observable angular distribution is broad enough to be readily detected by the next-generation $\gamma$-ray telescopes, and, possibly, by some of the currently operating ones. To our knowledge, these results have never been reported before. We have discussed how different choice of parameters (such as source redshift, primary proton energy and angular distribution, extragalactic magnetic field and extragalactic background light models) would change the observable spectra. We have also provided a qualitative discussion of the main stages of cascade development and the effects of additional processes.

This study could significantly facilitate future axion-like particle searches in the optically thick region of blazar spectra. In addition, our work might be useful in extreme $\mathrm{TeV}$ blazar studies as well as in several related branches of $\gamma$-ray astronomy, including extragalactic background light and extragalactic magnetic field measurements.

\section{ACKNOWLEDGEMENTS}

We are greatly indebted to Dr. S. Hackstein for guiding us through the CRPropa3 simulations and to E.I. Podlesnyi for running some of these simulations while the first author of this paper was on a duty journey. We are grateful to the members of the University of Tokyo, Institute for Cosmic Ray Research (ICRR) Cherenkov cosmic $\gamma$-ray group, especially to Prof. M. Teshima. We acknowledge helpful discussions with Dr. O.E. Kalashev, Dr. G.I. Rubtsov, Prof. S.V. Troitsky, Dr. A.V. Uryson, as well as with some of the participants of the Extreme-2019 and TeVPA-2019 conferences. We are grateful to the anonymous referee for the timely review, and for the suggestion to consider the role of triplet pair production, double pair production, and interactions on the universal radio background. This work was supported by the Russian Science Foundation (RSF) (project no. 18-72-00083).

\section{DATA AVAILABILITY STATEMENT}

The data underlying this article will be shared on reasonable request to the corresponding author.

\section{REFERENCES}

Aab A., Abreu P., Aglietta M., Ahn E., Samarai I. A., et al. 2014, Physical Review D, 90, 122006

Aab A., Abreu P., Aglietta M., Samarai I. A., Albuquerque I., et al. 2017a, Physical Review D, 96, 122003

Aab A., Abreu P., Aglietta M., Samarai I. A., Albuquerque I. F. M., et al. 2017b, Science, 357, 1266

Abdalla H., Abramowski A., Aharonian F., Benkhali F. A., Akhperjanian A. G., et al. 2017, Astronomy \& Astrophysics, 606, A59

Abdollahi S., Ackermann M., Ajello M., Atwood W. B., Baldini L., Ballet J., et al. 2018, Science, 362, 1031

Abeysekara A. U., Albert A., Alfaro R., Alvarez C., Alvarez J. D., et al. 2017, The Astrophysical Journal, 843, 39

Abeysekara A. U., et al., 2019, The Astrophysical Journal, 885, 150

Abraham J., Abreu P., Aglietta M., Ahn E. J., Allard D., et al. 2010, Physical Review Letters, 104, 091101

Abramowski A., Aharonian F., Benkhali F. A., Akhperjanian A. G., Angüner E., et al. 2014, Astronomy \& Astrophysics, 562, A145

Acciari V. A., Ansoldi S., Antonelli L. A., Engels A. A., Baack D., et al. 2019a, Monthly Notices of the Royal Astronomical Society, 486, 4233

Acciari V. A., Ansoldi S., Antonelli L. A., Engels A. A., Baack D., et al. 2019b, Monthly Notices of the Royal Astronomical Society, 490, 2284

Acciari V. A., et al., 2020, The Astrophysical Journal Supplement Series, 247, 16

Acharya B., Actis M., Aghajani T., Agnetta G., Aguilar J., et al. 2013, Astroparticle Physics, 43, 3

Ackermann M., Ajello M., Allafort A., Schady P., Baldini L., et al. 2012, Science, 338, 1190

Ackermann M., Ajello M., Baldini L., Ballet J., Barbiellini G., et al. 2018a, The Astrophysical Journal Supplement Series, 237, 32

Ackermann M., et al., 2018b, The Astrophysical Journal Supplement Series, 237, 32

Actis M., Agnetta G., Aharonian F., A A., Aleksic J., et al. 2011, Experimental Astronomy, 32, 193

Ade P. A. R., Aghanim N., Arnaud M., Ashdown M., Aumont J., et al. 2016, Astronomy \& Astrophysics, 594, A13

Aghanim N., et al., 2018 (arXiv: 1807.06209)

Aharonian F. A., Coppi P. S., Voelk H. J., 1994, The Astrophysical Journal, 423, L5

Aharonian F. A., Akhperjanian A. G., Barrio J. A., Bernlöhr K., Bolz O., et al. 2001, Astronomy \& Astrophysics, 366, 746

Aharonian F., Akhperjanian A. G., Bazer-Bachi A. R., Beilicke M., Benbow W., et al. 2006, Nature, 440, 1018

Aharonian F., Akhperjanian A. G., Bazer-Bachi A. R., Beilicke M., Benbow W., et al. 2007a, Astronomy \& Astrophysics, 470, 475

Aharonian F., Akhperjanian A. G., de Almeida U. B., Bazer-Bachi A. R., Behera B., et al. 2007b, Astronomy \& Astrophysics, 473, L25

Aharonian F., Akhperjanian A. G., de Almeida U. B., Bazer-Bachi A. R., Behera B., et al. 2007c, Astronomy \& Astrophysics, 475, L9

Aharonian F. A., Kelner S. R., Prosekin A. Y., 2010, Physical Review D, 82,043002

Aleksić J., Ansoldi S., Antonelli L., Antoranz P., Babic A., et al. 2016a, Astroparticle Physics, 72, 61

Aleksić J., Ansoldi S., Antonelli L., Antoranz P., Babic A., et al. 2016b, Astroparticle Physics, 72, 76

Aliu E., Archambault S., Arlen T., Aune T., Behera B., et al. 2014, The Astrophysical Journal, 782, 13

Alves Batista R., et al., 2016, Journal of Cosmology and Astroparticle Physics, 2016, 038

Alves Batista R., Shin M.-S., Devriendt J., Semikoz D., Sigl G., 2017, Physical Review D, 96

Alves Batista R., et al., 2019, Frontiers in Astronomy and Space Sciences, 6

Archer A., Benbow W., Bird R., Brose R., Buchovecky M., et al. 2018, The Astrophysical Journal, 862, 41

Arlen T. C., Vassilev V. V., Weisgarber T., Wakely S. P., Shafi S. Y., 2014, The Astrophysical Journal, 796, 18 
Atwood W. B., Abdo A. A., Ackermann M., Althouse W., Anderson B., et al. 2009, The Astrophysical Journal, 697, 1071

Bai X., et al., 2019 (arXiv: 1905.02773)

Baklagin S. A., Dzhatdoev T. A., Kircheva A. P., Lukshin A. A., Khalikov E. V., 2018, Physics of Particles and Nuclei, 49, 90

Berezinskii V. S., Grigor'eva S. I., 1988, Astronomy \& Astrophysics, 199, 1

Berezinsky V., Kalashev O., 2016, Physical Review D, 94, 023007

Berezinsky V. S., Smirnov A. Y., 1975, Astrophysics and Space Science, 32,461

Berezinsky V., Gazizov A., Grigorieva S., 2006, Physical Review D, 74, 043005

Berezinsky V., Gazizov A., Kachelrieß M., Ostapchenko S., 2011, Physics Letters B, 695, 13

Biasuzzi B., Hervet O., Williams D. A., Biteau J., 2019, Astronomy \& Astrophysics, 627, A110

Biermann P. L., Strittmatter P. A., 1987, The Astrophysical Journal, 322, 643

Biteau J., Williams D. A., 2015, The Astrophysical Journal, 812, 60

Biteau J., et al., 2020, Nature Astronomy, 4, 124

Blytt M., Kachelriess M., Ostapchenko S., 2019 (arXiv: 1909.09210)

Bonnefoy S., Ashton T., Backes M., Balzer A., Berge D., Brun F., et al. 2018, in Proceedings of 35th International Cosmic Ray Conference PoS(ICRC2017). Sissa Medialab, doi:10.22323/1.301.0805

Bonnoli G., Tavecchio F., Ghisellini G., Sbarrato T., 2015, Monthly Notices of the Royal Astronomical Society, 451, 611

Broderick A. E., Chang P., Pfrommer C., 2012, The Astrophysical Journal, 752, 22

Brown R. W., Hunt W. F., Mikaelian K. O., Muzinich I. J., 1973, Physical Review D, 8, 3083

Brunetti G., Vazza F., 2020, Physical Review Letters, 124, 051101

Bulgarelli A., Fioretti V., Parmiggiani N., Verrecchia F., Pittori C., et al. 2019, Astronomy \& Astrophysics, 627, A13

Chang P., Broderick A. E., Pfrommer C., Puchwein E., Lamberts A., Shalaby M., 2014, The Astrophysical Journal, 797, 110

Costamante L., et al., 2001, Astronomy \& Astrophysics, 371, 512

Costamante L., Bonnoli G., Tavecchio F., Ghisellini G., Tagliaferri G., Khangulyan D., 2018, Monthly Notices of the Royal Astronomical Society, 477, 4257

Cui S., Liu Y., Liu Y., Ma X., 2014, Astroparticle Physics, 54, 86

Das S., Kang H., Ryu D., Cho J., 2008, The Astrophysical Journal, 682, 29

Das S., Gupta N., Razzaque S., 2020, The Astrophysical Journal, 889, 149

De Angelis A., Roncadelli M., Mansutti O., 2007, Physical Review D, 76, 121301

De Angelis A., Galanti G., Roncadelli M., 2011, Physical Review D, 84, 105030

De Angelis A., Tatischeff V., Grenier I., McEnery J., Mallamaci M., et al. 2018, Journal of High Energy Astrophysics, 19, 1

Dermer C. D., Razzaque S., 2010, The Astrophysical Journal, 724, 1366

Dermer C. D., Cavadini M., Razzaque S., Finke J. D., Chiang J., Lott B., 2011, The Astrophysical Journal, 733, L21

Dolag K., Grasso D., Springel V., Tkachev I., 2005, Journal of Cosmology and Astroparticle Physics, 2005, 009

Dolgoshein B., Lebedenko V., Rodionov B., 1970, Soviet Journal of Experimental and Theoretical Physics Letters, 11, 351

Domínguez A., et al., 2011, Monthly Notices of the Royal Astronomical Society, 410,2556

Dzhatdoev T. A., 2015, Journal of Physics: Conference Series, 632, 012035

Dzhatdoev T., Podlesnyi E., 2019, Astroparticle Physics, 112, 1

Dzhatdoev T. A., Khalikov E. V., Kircheva A. P., Lyukshin A. A., 2017, Astronomy \& Astrophysics, 603, A59

Dzhatdoev T., Khalikov E., Podlesnyi E., Telegina A., 2019, Journal of Physics: Conference Series, 1181, 012049

Eichmann B., 2019, Journal of Cosmology and Astroparticle Physics, 2019, 009

Ellien A., Durret F., Adami C., Martinet N., Lobo C., Jauzac M., 2019, Astronomy \& Astrophysics, 628, A34

Essey W., Kalashev O. E., Kusenko A., Beacom J. F., 2010, Physical Review Letters, 104
Essey W., Kalashev O., Kusenko A., Beacom J. F., 2011, The Astrophysical Journal, 731, 51

Finke J. D., Reyes L. C., Georganopoulos M., Reynolds K., Ajello M., Fegan S. J., McCann K., 2015, The Astrophysical Journal, 814, 20

Fitoussi T., Belmont R., Malzac J., Marcowith A., Cohen-Tanugi J., Jean P., 2017, Monthly Notices of the Royal Astronomical Society, 466, 3472

Franceschini A., Rodighiero G., 2017, Astronomy \& Astrophysics, 603, A34

Franceschini A., Rodighiero G., 2018, Astronomy \& Astrophysics, 614, C1

Franceschini A., Rodighiero G., Vaccari M., 2008, Astronomy \& Astrophysics, 487, 837

Franceschini A., Foffano L., Prandini E., Tavecchio F., 2019, Astronomy \& Astrophysics, 629, A2

Galanti G., Roncadelli M., 2018, Physical Review D, 98, 043018

Galanti G., Tavecchio F., Roncadelli M., Evoli C., 2019, Monthly Notices of the Royal Astronomical Society, 487, 123

Galper A., Adriani O., Aptekar R., Arkhangelskaja I., Arkhangelskiy A., et al. 2013, Advances in Space Research, 51, 297

Gehrels N., Chincarini G., Giommi P., Mason K. O., Nousek J. A., et al. 2004, The Astrophysical Journal, 611, 1005

Gilmore R. C., Somerville R. S., Primack J. R., Domínguez A., 2012, Monthly Notices of the Royal Astronomical Society, 422, 3189

Govoni F., Orrù E., Bonafede A., Iacobelli M., Paladino R., et al. 2019, Science, 364, 981

Greisen K., 1966, Physical Review Letters, 16, 748

Gros P., et al., 2018, Astroparticle Physics, 97, 10

Gruzinov A., 2018 (arXiv: 1808.00041)

Hackstein S., Vazza F., Brüggen M., Sorce J. G., Gottlöber S., 2018, Monthly Notices of the Royal Astronomical Society, 475, 2519

Hackstein S., Brüggen M., Vazza F., Gaensler B. M., Heesen V., 2019, Monthly Notices of the Royal Astronomical Society, 488, 4220

Harari D., Mollerach S., Roulet E., 2016, Physical Review D, 93, 063002

Heald G., Mao S., Vacca V., Akahori T., Damas-Segovia A., et al. 2020, Galaxies, 8, 53

Hillas A. M., 1984, Annual Review of Astronomy and Astrophysics, 22, 425

Hinton J., 2004, New Astronomy Reviews, 48, 331

Horns D., Meyer M., 2012, Journal of Cosmology and Astroparticle Physics, 2012, 033

Hunter S. D., et al., 2014, Astroparticle Physics, 59, 18

Inoue Y., Inoue S., Kobayashi M. A. R., Makiya R., Niino Y., Totani T., 2013, The Astrophysical Journal, 768, 197

Jansen F., et al., 2001, Astronomy \& Astrophysics, 365, L1

Jarp S., Mork K. J., 1973, Physical Review D, 8, 159

Kachelrieß M., Ostapchenko S., Tomàs R., 2012, Computer Physics Communications, 183, 1036

Kalashev O. E., Kido E., 2015, Journal of Experimental and Theoretical Physics, 120,790

Kalashev O. E., Kuzmin V. A., Semikoz D. V., 2001, Modern Physics Letters A, 16, 2505

Kalashev O. E., Semikoz D. V., Sigl G., 2009, Physical Review D, 79, 063005

Kartavtsev A., Raffelt G., Vogel H., 2017 , Journal of Cosmology and Astroparticle Physics, 2017, 024

Kempf A., Kilian P., Spanier F., 2016, Astronomy \& Astrophysics, 585, A132

Khangulyan D., Aharonian F. A., Kelner S. R., 2014, The Astrophysical Journal, 783, 100

Kneiske T. M., Dole H., 2010, Astronomy and Astrophysics, 515, A19

Korochkin A. A., Rubtsov G. I., 2018, Monthly Notices of the Royal Astronomical Society, 481, 557

Korochkin A., Rubtsov G., Troitsky S., 2019, Journal of Cosmology and Astroparticle Physics, 2019, 002

Krennrich F., Bond I., Boyle P., Bradbury S., Buckley J., et al. 2004, New Astronomy Reviews, 48, 345

Lee S., 1998, Physical Review D, 58

Lombardi S., et al., 2020, Astronomy \& Astrophysics, 634, A22 
Mastichiadis A., Marscher A. P., Brecher K., 1986, The Astrophysical Journal, 300, 178

Mastichiadis A., Protheroe R. J., Szabo A. P., 1994, Monthly Notices of the Royal Astronomical Society, 266, 910

McEnery J., Barrio J. A., Agudo I., Ajello M., Álvarez J.-M., et al. 2019 (arXiv: 1907.07558)

Medina Tanco G. A., 1998, The Astrophysical Journal, 505, L79

Menzler U., Schlickeiser R., 2015, Monthly Notices of the Royal Astronomical Soct 448, 3405

Merloni A., Predehl P., Becker W., Böhringer H., Boller T., et al. 2012 (arXiv: 1209.3114)

Miniati F., Elyiv A., 2013, The Astrophysical Journal, 770, 54

Montanino D., Vazza F., Mirizzi A., Viel M., 2017, Physical Review Letters, 119, 101101

Müller G., 2016, Journal of Cosmology and Astroparticle Physics, 2016, 025

Murase K., Dermer C. D., Takami H., Migliori G., 2012, The Astrophysical Journal, 749, 63

Nandra K., Barret D., Barcons X., Fabian A., den Herder J.-W., et al. 2013 (arXiv: 1306.2307)

Neronov A., Semikoz D. V., 2009, Physical Review D, 80, 123012

Neronov A., Vovk I., 2010, Science, 328, 73

Niţu I., Bevins H., Bray J., Scaife A., 2021, Astroparticle Physics, 126, 102532

Park N., 2016, in Proceedings of The 34th International Cosmic Ray Conference — PoS(ICRC2015). Sissa Medialab, doi:10.22323/1.236.0771

Primack J. R., Bullock J. S., Somerville R. S., 2005, AIP Conference Proceedings, 745, 23

Protheroe R. J., 1986, Monthly Notices of the Royal Astronomical Society, 221, 769

Protheroe R., Biermann P., 1996, Astroparticle Physics, 6, 45

Protheroe R. J., Stanev T., 1993, Monthly Notices of the Royal Astronomical Society, 264, 191

Rachen J. P., Biermann P. L., 1993, Astronomy \& Astrophysics, 272, 161

Raffelt G., Stodolsky L., 1988, Physical Review D, 37, 1237

Rubbia C., 1977, CERN-EP-INT-77-08

Rubbia C., et al., 2011, Journal of Instrumentation, 6, P07011

Rubtsov G. I., Troitsky S. V., 2014, Soviet Journal of Experimental and Theoretical Physics Letters, 100,355

Ryu D., Kang H., Biermann P. L., 1998, Astronomy \& Astrophysics, 335,19

Sánchez-Conde M. A., Paneque D., Bloom E., Prada F., Domínguez A., 2009, Physical Review D, 79, 123511

Schlickeiser R., Ibscher D., Supsar M., 2012, The Astrophysical Journal, 758,102

Sigl G., Miniati F., Enßlin T. A., 2004, Physical Review D, 70, 043007

Sironi L., Giannios D., 2014, The Astrophysical Journal, 787, 49

Stecker F. W., Scully S. T., Malkan M. A., 2016, The Astrophysical Journal, 827,6

Strong A., 2018, Nuclear and Particle Physics Proceedings, 297-299, 165

Tavani M., Barbiellini G., Argan A., Boffelli F., Bulgarelli A., et al. 2009, Astronomy \& Astrophysics, 502, 995

Tavecchio F., Ghisellini G., Foschini L., Bonnoli G., Ghirlanda G., Coppi P., 2010, Monthly Notices of the Royal Astronomical Society: Letters, 406, L70

Taylor A. M., Vovk I., Neronov A., 2011, Astronomy \& Astrophysics, 529, A144

The CTA Consortium 2018, Science with the Cherenkov Telescope Array. World Scientific, doi:10.1142/10986

Tian Z., Wang Z., Liu Y., Guo Y., Ma X., Hu H., 2018, Astroparticle Physics, 99, 43

Uryson A. V., 1998, Journal of Experimental and Theoretical Physics, 86, 213

Vacca V., Murgia M., Govoni F., Loi F., Vazza F., et al. 2018, Monthly Notices of the Royal Astronomical Society, 479, 776

Vafin S., Rafighi I., Pohl M., Niemiec J., 2018, The Astrophysical Journal, 857,43
Vafin S., Deka P. J., Pohl M., Bohdan A., 2019, The Astrophysical Journal, 873,10

Vazza F., Brüggen M., Gheller C., Hackstein S., Wittor D., Hinz P. M., 2017, Classical and Quantum Gravity, 34, 234001

Waxman E., Coppi P., 1996, The Astrophysical Journal, 464, L75

Wilkes B., Tucker W., eds, 2019, The Chandra X-ray Observatory. 25143433, IOP Publishing, doi:10.1088/2514-3433/ab43dc

ettsepin G. T., Kuz'min V. A., 1966, Soviet Journal of Experimental and Theoretical Physics Letters, 4, 78

Zheng Y. G., Yang C. Y., Kang S. J., 2015, Astronomy \& Astrophysics, 585, A8 


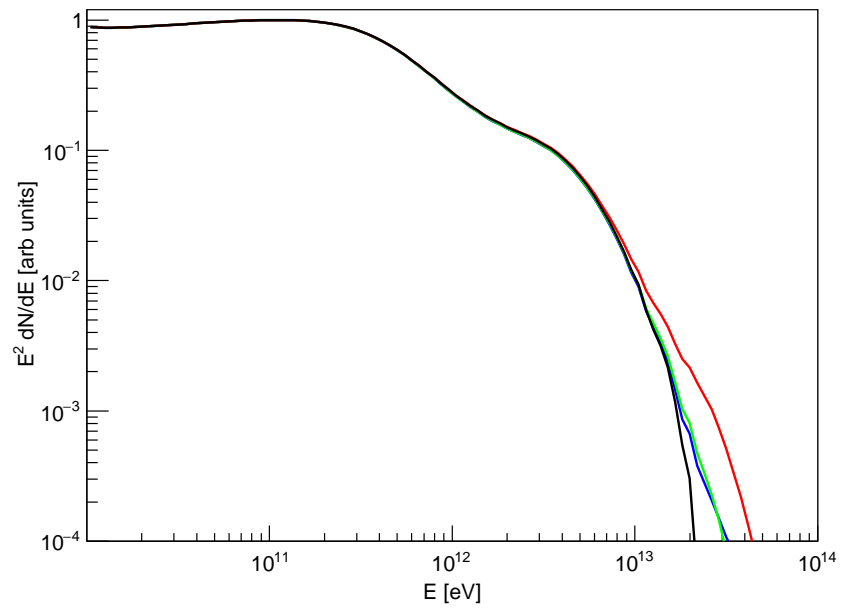

Figure A1. Average observable SEDs for the KD10 EBL model option and the following numbers of trajectories: 10 (black curve), 100 (red curve), $10^{3}$ (green curve), $10^{4}$ (blue curve).

\section{APPENDIX A: THE IMPACT OF FLUCTUATIONS ON THE OBSERVABLE SPECTRA}

The observable spectrum in the framework of the MHCM depends on the EGMF structure, in particular, on the number of proton trajectories used in our study. In Fig. A1 we show the observable SEDs averaged over various numbers of proton trajectories. We conclude that the statistics of $10^{2}$ trajectories is sufficient to obtain a stable enough average observable spectrum for $E<10 \mathrm{TeV}$, and the use of $10^{3}$ trajectories already allows one to robustly determine the shape of the spectrum for $E<30 \mathrm{TeV}$.

In addition, we have calculated the observable SEDs for $10^{2}$ individual proton trajectories to demonstrate the impact of fluctuations on the shape of the observable spectrum (see Fig. A2). The blue-to-red gradient of colors gives a visual representation to the values of the ratios of different individual SEDs at two benchmark energies: $10 \mathrm{TeV}$ and $100 \mathrm{GeV}$. Blue colors represent lower ratios, while red colors - higher ones. We note that at $E=5 \mathrm{TeV}$ the maximal difference between the flux corresponding to $95 \%$ of the trajectories is limited to the factor of 2.5 .

\section{APPENDIX B: DISTRIBUTION OF THE CRITICAL DISTANCE}

In Fig. B1 we present a histogram of a distribution on "the critical distance" $L_{c r}$ (see Subsect 4.1 for definition of this parameter). The normalization of this histogram corresponds to the case of discrete probability density function, i.e. $\sum_{i=0}^{N} F_{i}=1$ with summation over the bins of the histogram, with the $N$ th bin containing the largest value of $L_{c r}$.

\section{APPENDIX C: SPECTRA WITH AND WITHOUT TPP, URB, DPP}

In Fig. $\mathrm{C} 1$ we present histograms of the observable spectra of $\gamma$-rays and electrons calculated with the CRPropa3 code for the case of the primary $\gamma$-rays and electrons with the energy of $1 \mathrm{EeV}$ and the distance between the source and the observer of $10 \mathrm{Mpc}$. $\gamma$-ray spectra obtained with the account of the interactions on the

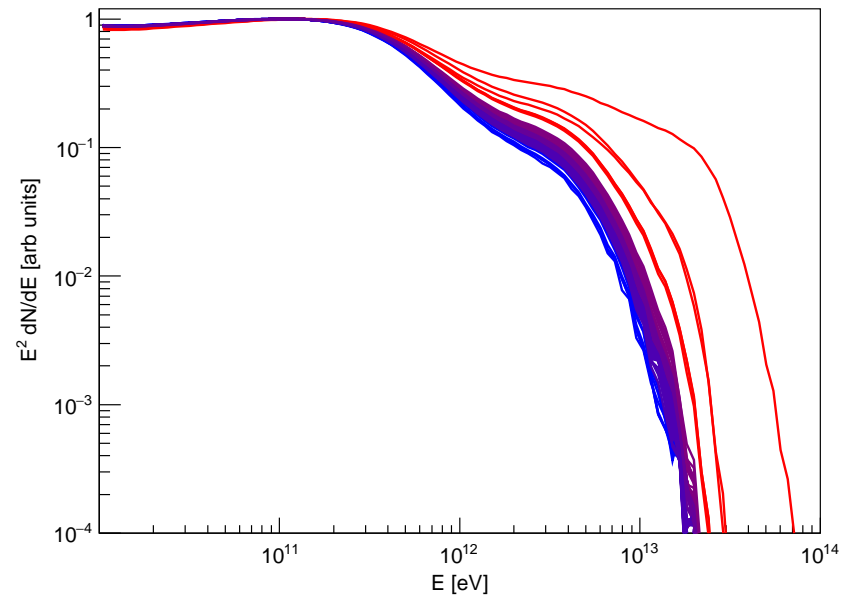

Figure A2. Observable SEDs for the KD10 EBL model option and $10^{2}$ individual trajectories; colors represent the ratios of individual SEDs at 10 $\mathrm{TeV}$ and $100 \mathrm{GeV}$.

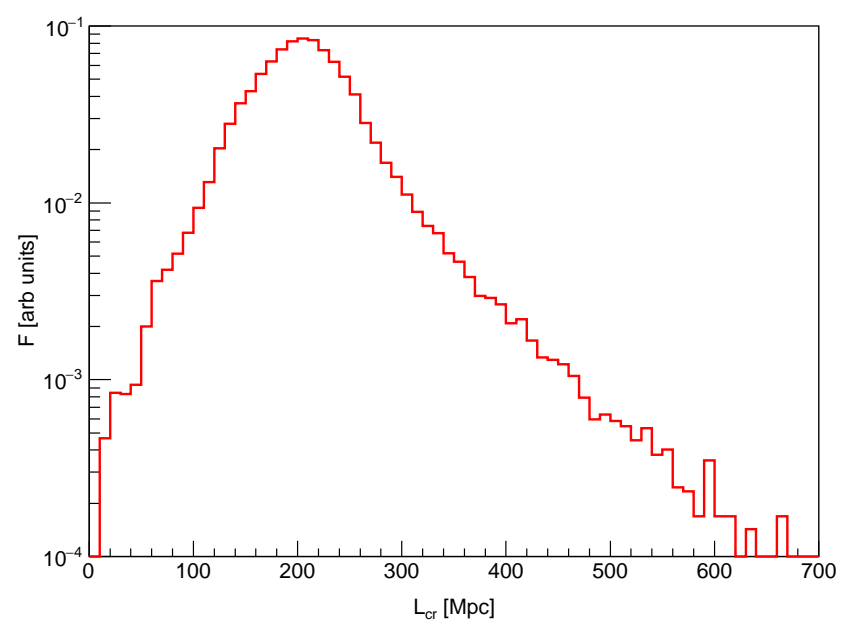

Figure B1. Histogram of the critical distance $L_{c r}$ distribution (red curve).

URB and the TPP and DPP processes have a marked energy cutoff at $100 \mathrm{TeV}$. Moreover, in this case the intensity of observable electrons is subdominant with respect to $\gamma$-rays. On the contrary, when the interactions on the URB and the TPP and DPP processes are neglected, observable $\gamma$-ray and electron spectra appear to be much harder.

To conclude, for the case of $E_{0}=1 \mathrm{EeV}$ the difference between the observable spectra after the propagated distance of 10 Mpc with and without account of URB, TPP, and DPP is significant (cf. Mastichiadis et al. (1994): "triplet production is particularly important (...) for propagation over small path length”), but for the propagation distances much larger than $10 \mathrm{Mpc}$ the observable spectra are almost the same (again, cf. Mastichiadis et al. (1994): TPP "has no effect on saturated cascades").

This paper has been typeset from a $\mathrm{T}_{\mathrm{E}} \mathrm{X} / \mathrm{L} \mathrm{AT} \mathrm{E} \mathrm{X}$ file prepared by the author. 


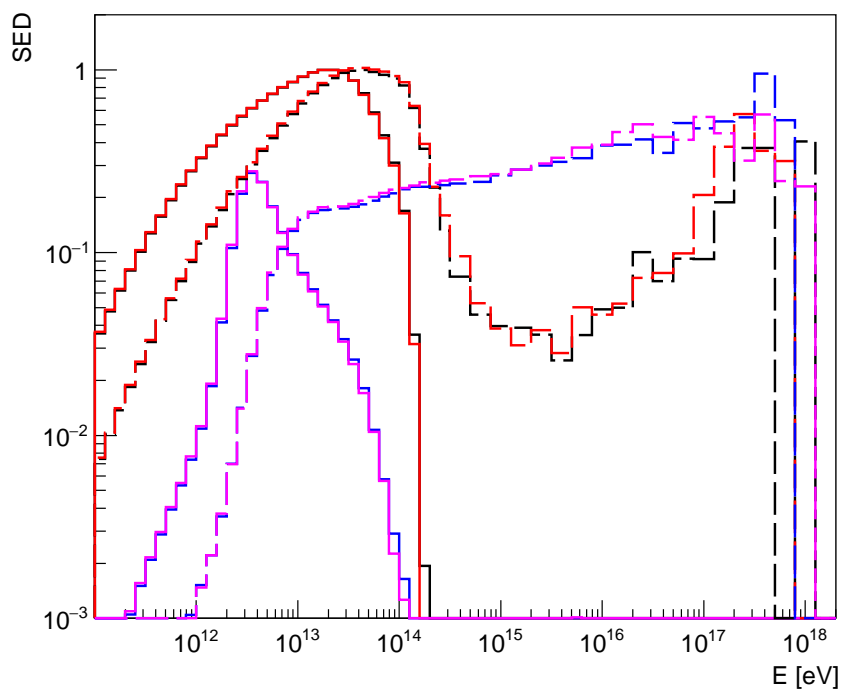

Figure C1. Observable spectra of $\gamma$-rays and electrons with the account of the interactions on the URB and the TPP and DPP processes (solid curves) as well as the same spectra neglecting these processes (dashed curves). Black histograms denote observable spectra of $\gamma$-rays from primary $\gamma$-rays, red histograms - spectra of $\gamma$-rays from primary electrons, blue histograms - spectra of electrons from primary $\gamma$-rays, magenta histograms - spectra of electrons from primary electrons. 